\title{
Identification of Potential Water-Resources- Monitoring Sites in the Croton Reservoir System, Southeastern New York
}

By Kristin S. Linsey, Stephen W. Wolcott, and Nancy B. Schoonmaker

U.S. GEOLOGICAL SURVEY

Open-File Report 97-638

Prepared in cooperation with the New York City Department of

Environmental Protection

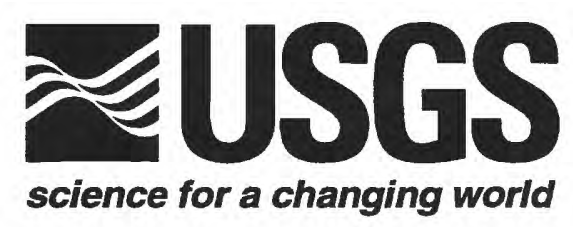

Albany, New York 1999 


\section{U.S. DEPARTMENT OF THE INTERIOR BRUCE BABBITT, Secretary}

\section{U.S. GEOLOGICAL SURVEY}

Charles G. Groat, Director

For addtional information write to:

District Chief

U.S. Geological Survey

425 Jordan Road

Troy, New York 12180
Copies of this report can be purchased from:

U.S. Geological Survey

Branch of Information Services

Box 25286

Denver, CO 80225-0286 


\section{CONTENTS}

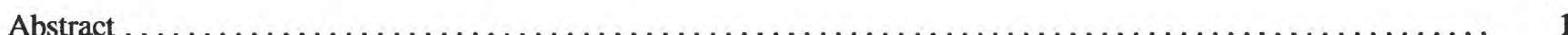

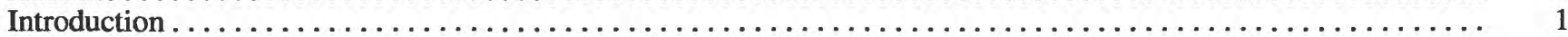

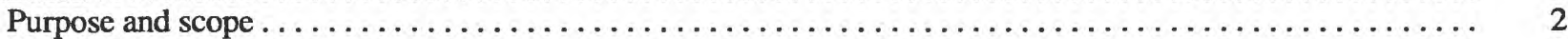

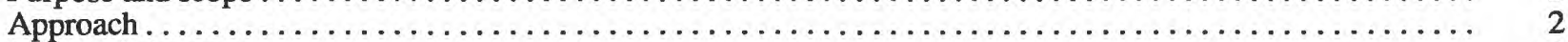

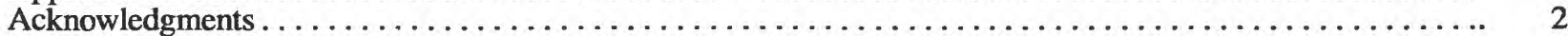

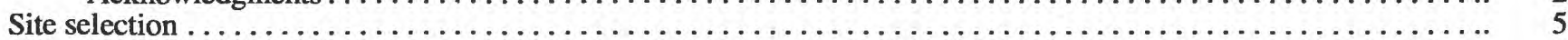

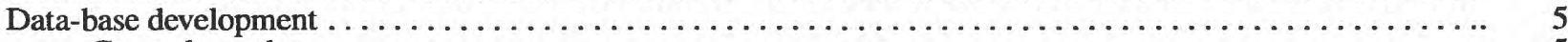

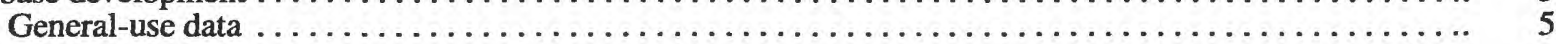

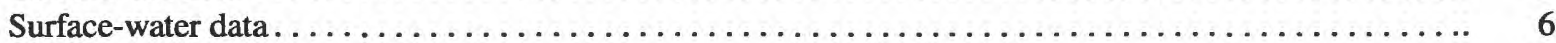

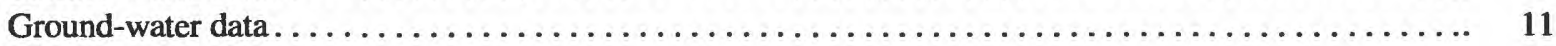

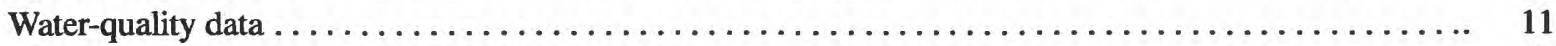

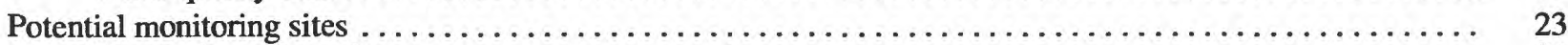

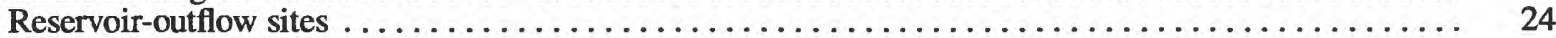

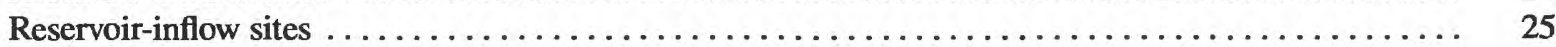

Ground-water-monitoring areas. .................................... 29

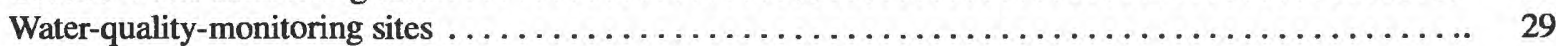

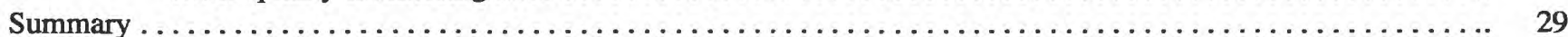

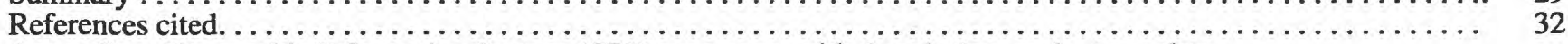

Appendix - Geographic Information System (GIS) coverages, with description, scale, type, data source, and development procedure for each coverage used in selection of monitoring sites . . . . . . .

\section{FIGURES}

Figures 1-10. Maps of the Croton Reservoir system, N.Y. showing:

1. Location and major geographic features. . . . . . . . . . . . . . . . . . . . . . . . . . .

2. 1990 population, by census block. . . . . . . . . . . . . . . . . . . . . . . . . . . . .

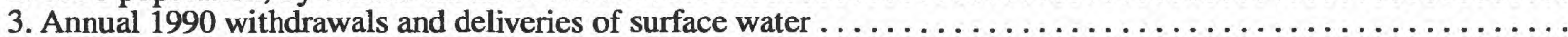

4. Drainage divides and surface-water data-collection sites established by the U.S. Geological Survey. . . . . . . . .

5. A. Surficial geology and location of ground-water withdrawal sites for public-supply wells and wells with potential yields of more than 50 gallons per minute from

surficial deposits. . . . . . . . . . . . . . . . . . . . . . . . . . . . . . . . .

B. Bedrock geology and location of ground-water withdrawal sites for public-supply wells finished in bedrock and wells with potential yields of at least 50 gallons per minute from bedrock $\ldots \ldots \ldots \ldots \ldots \ldots$

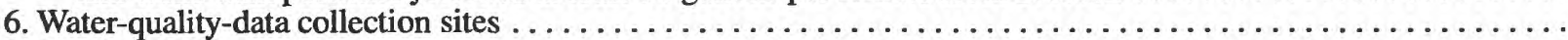

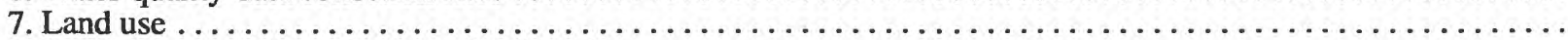

8. Potential reservoir-outflow monitoring sites. . . . . . . . . . . . . . . . . . . . . . . .

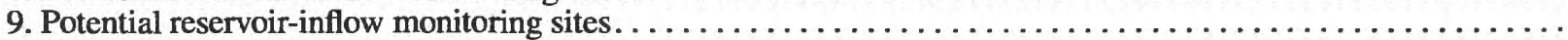

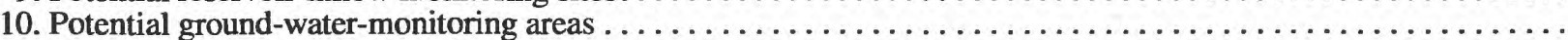

\section{TABLES}

1. Types of data collected at surface-water data-collection sites. $\ldots \ldots \ldots \ldots \ldots \ldots \ldots \ldots \ldots \ldots \ldots \ldots$

2. Data on ground-water withdrawal sites for public-water supply and selected

high-yielding wells.

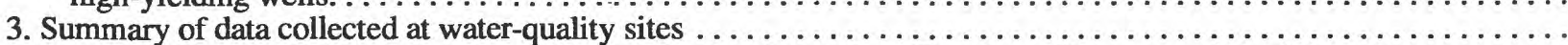

4. Sites that are potential point-sources of contamination in southern Dutchess, Putnam, and northern

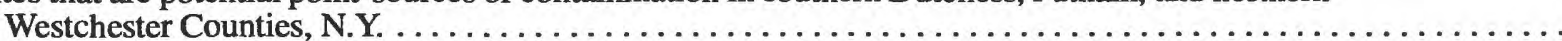

5. Monitoring categories to which each GIS coverage was applied in site-selection process in the

Croton Reservoir system . . . . . . . . . . . . . . . . . . . . . . . . . . . . . . . .

6. Potential reservoir outflow monitoring sites in the Croton Reservoir system . . . . . . . . . . . . . . .

7. Sites selected for reservoir-inflow monitoring in the Croton Reservoir system . . . . . . . . . . . . . . .

8. Potential ground-water-monitoring areas in the Croton Reservoir System . . . . . . . . . . . . . . . . 
For the convenience of readers who prefer metric (International System) units to the inch pound units used in this report, the following conversion factors may be used:

Multiply By obtain

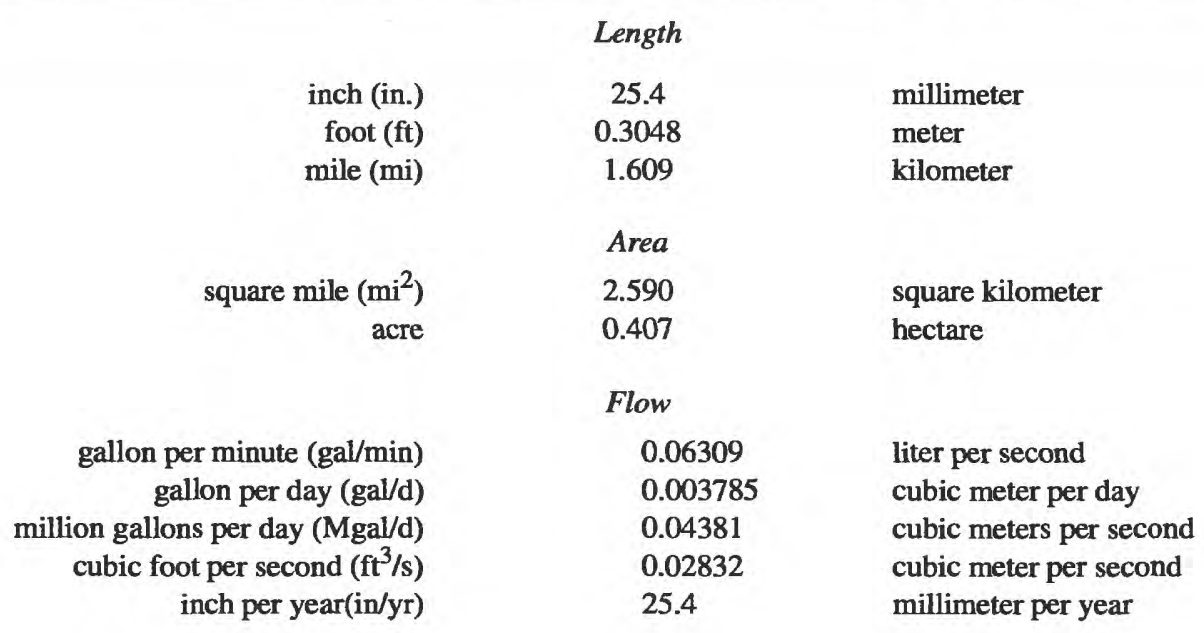

Sea level: In this report, "sea level" refers to the National Geodetic Vertical Datum of 1929 (NGVD of 1929) - a geodetic datum derived from a general adjustment of the first-order level nets of both the United States and Canada, formerly called Sea Level Datum of 1929. 


\title{
IDENTIFICATION OF POTENTIAL WATER-RESOURCES-MONITORING SITES IN THE CROTON RESERVOIR SYSTEM, SOUTHEASTERN NEW YORK
}

\author{
By Kristin S. Linsey, Stephen W. Wolcott, and Nancy B. Schoonmaker
}

\section{ABSTRACT}

Urbanization has led to the deterioration of water quality within the Croton Reservoir system, a 374-square-mile watershed with 12 reservoirs in southeastern New York that supply 10 percent of New York City's water. Water-resources managers need hydrologic data to develop plans to protect and conserve the water resources of this watershed. In 1990, the U.S. Geological Survey in cooperation with New York City Department of Environmental Protection (NYCDEP) began a 2-year program to establish water-resources data-collection sites that, together, would provide managers with the information needed to monitor and evaluate the water resources of the Croton Reservoir System.

Sites for the water-resources monitoring network in the Croton Reservoir system were subjectively selected with the aid of a Geographic Information System (GIS). The site-selection process entailed (1) developing a GIS data base of long-term and current water-resources data, and (2) using the GIS data base to identify potential sites for (A) water sampling and (B) measurement of surface-water flow into and out of selected reservoirs and (C) monitoring ground-water levels. Use of a GIS to store the data collected for this study aided in the analysis of the data during the site-selection process and could be used in a similar manner to establish water-resources-monitoring networks in other watersheds.

The data base that was developed in this study contains 13 GIS coverages and 2 related tables. Data were obtained from the NYCDEP, the New York State Department of Environmental Conservation, and the U.S. Geological Survey
(USGS) National Water Information System (NWIS) data bases. The scale of the source maps ranged from $1: 24,000$ to $1: 250,000$.

Three groups of potential monitoring sites were identified: (1) reservoir-outflow-monitoring sites, (2) reservoir-inflow-monitoring sites, and (3) ground-water-monitoring areas. Eight reservoir-outflow-monitoring sites were identified to obtain discharge and water-quality data for eight of the twelve reservoirs within the system and nine reservoir-inflow-monitoring sites were selected to provide data on streamflow characteristics and chemical quality of water flowing into these reservoirs. Sixteen ground-water monitoring areas were selected to provide an overview of ground-water levels and chemical quality of ground water in the Croton reservoir system.

\section{INTRODUCTION}

Urbanization and changes in land use can alter the quality and quantity of water that flows from a given watershed. Expanding urbanization in the 374-mi2 Croton River basin (fig. 1) in southeastern New York has led to the deterioration of water quality in the Croton Reservoir system, which is managed by the City of New York and supplies 10 percent of the City's water, but could supply as much as 25 percent during periods of drought (Goldin, 1989). The water does not require filtration at present, but if current trends in population growth (fig. 2) and in waterquality deterioration continue, filtration and other costly types of treatment may be required. Waterresources managers need data on reservoir storage, streamflow, ground-water levels, and the chemical quality of ground water and surface water to monitor 
these resources and to develop plans to protect and conserve them.

In 1990, the U.S. Geological Survey (USGS), in cooperation with the New York City Department of Environmental Protection (NYCDEP), began a 2-year study to identify appropriate data-collection sites for monitoring the water resources of the Croton Reservoir system. This water-resources monitoring network would consist of three categories of sites-reservoiroutflow-measurement sites, reservoir-inflow-measurement sites, and ground-water-monitoring areas. Waterquality data would be collected at all sites in each of the three categories.

The main objective of the study was to subjectively establish the optimum number and location of sites needed to adequately monitor streamflow, ground-water levels, and chemical quality of surface water and ground water in the basin. Other objectives were to document the approach used for site selection and to develop data bases, through Geographical Information System (GIS) technology, for use during the study and in the future. The type of GIS data bases developed in this study could be used to help establish water-resources-monitoring networks in other watersheds. A total of 13 GIS coverages and 2 data tables were developed.

\section{Purpose and Scope}

This report describes (1) the categories of data that were collected, (2) which types of data from each category were used in the subjective site selection, and (3) the potential sites that were selected as appropriate for the water-resources monitoring network. Selected data that were compiled during this study are depicted on 10 maps that were generated with a GIS. The appendix summarizes the type and sources of data, the map scale, and the procedures used in the development of the 13 coverages and the 2 related data tables for this study.

\section{Approach}

Development of plans to protect and conserve the water resources of the Croton Reservoir system will require a variety of hydrologic data, including lake or reservoir outflows and inflows, ground-water levels, and chemical quality of surface water and ground water within the basin. Reservoir-outflow and -inflow data enable monitoring of changes in the reservoir storage and detection of changes in the chemical quality of their water. Ground-water data allow calculation of the availability and quality of ground water that contributes to baseflow throughout the watershed. Thus the data-collection sites would be selected in locations that would provide those types of data; sites also would be selected in areas that represent unique characteristics related to land use or bedrock geology, or with characteristics that are similar to those in other parts of the watershed, so information would be transferable to those areas.

The study was conducted in two stages. The first stage entailed collection of long-term and current data on population, bedrock and surficial geology, streamflow, ground-water levels, chemical quality of surface water and ground water, and land use. Sources of data included the New York City Department of Environmental Protection, the New York State Department of Environmental Conservation (NYSDEC), county agencies, and U.S. Geological Survey National Water Information System (NWIS) data bases. The scale of the maps from which data were collected ranged from $1: 24,000$ to $1: 250,000$. The data were incorporated into a GIS data base; similar types of data were merged into a single data set or table.

The second stage of the study entailed using the GIS data bases to subjectively identify potential datacollection sites for the water-resource-monitoring network. The primary sources of data that were used to help select reservoir-outflow sites included the locations of dams maintained by New York City, subbasin drainage boundaries, and surface-water diversions. The reservoir-inflow sites were selected from data bases showing subbasin drainage boundaries and the land-use practices within the study area, and the ground-watermonitoring areas were selected from the previously determined reservoir-inflow subbasin drainage boundaries and from surficial and bedrock geologic maps.

\section{Acknowledgments}

The NYCDEP provided water-quality data, reservoir-stage data, and diversions into and out of the Croton Reservoir system. The authors thank Eliot Schneiderman of the NYCDEP and Sam Wear of the Westchester County Planning Department for providing land-use data for significant parts of the study area. 


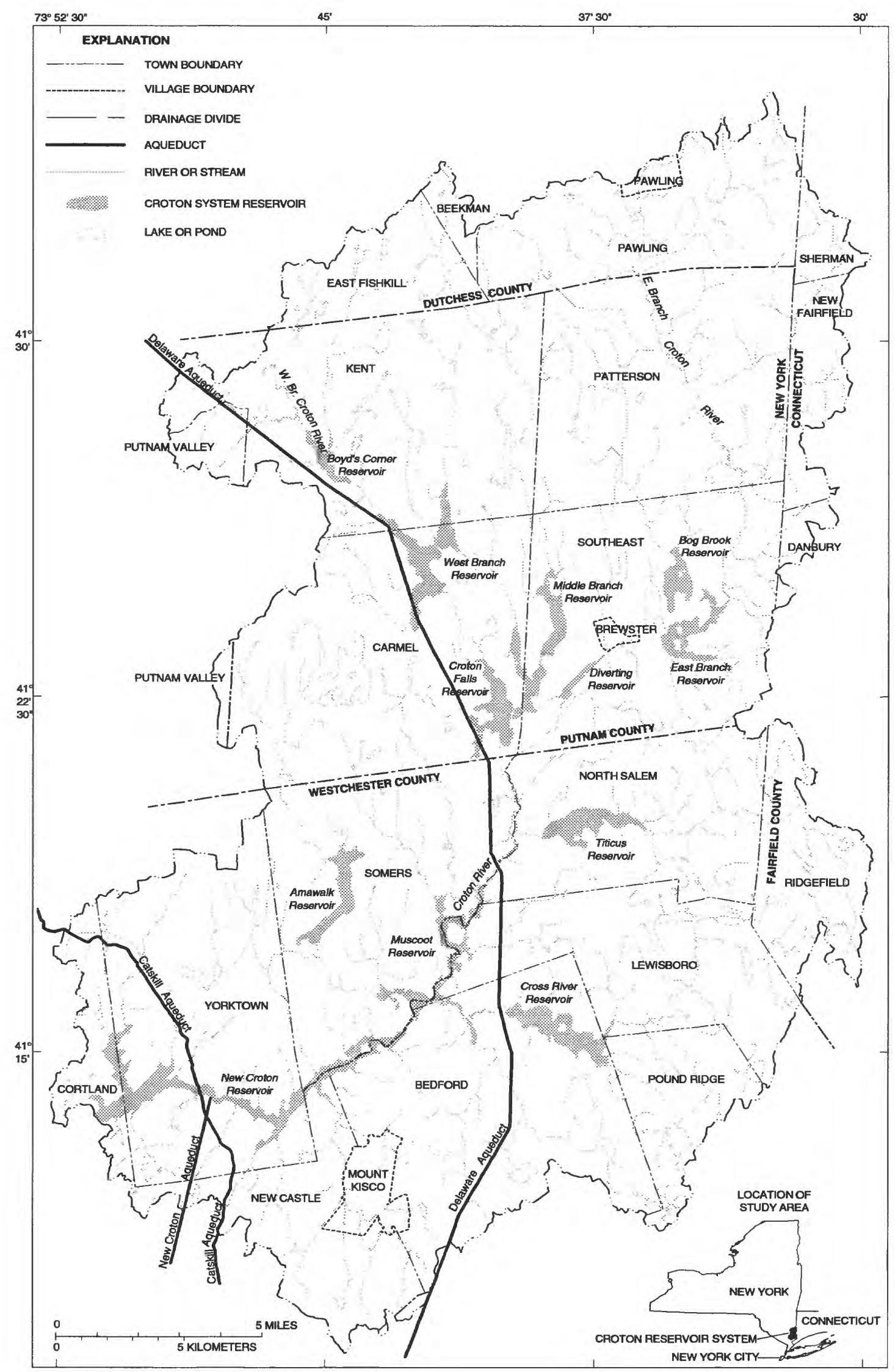

Base from U.S. Geological Survey digital data, 1:100,000, 1983

Figure 1. Location and major geographic features of the Croton Reservoir system 


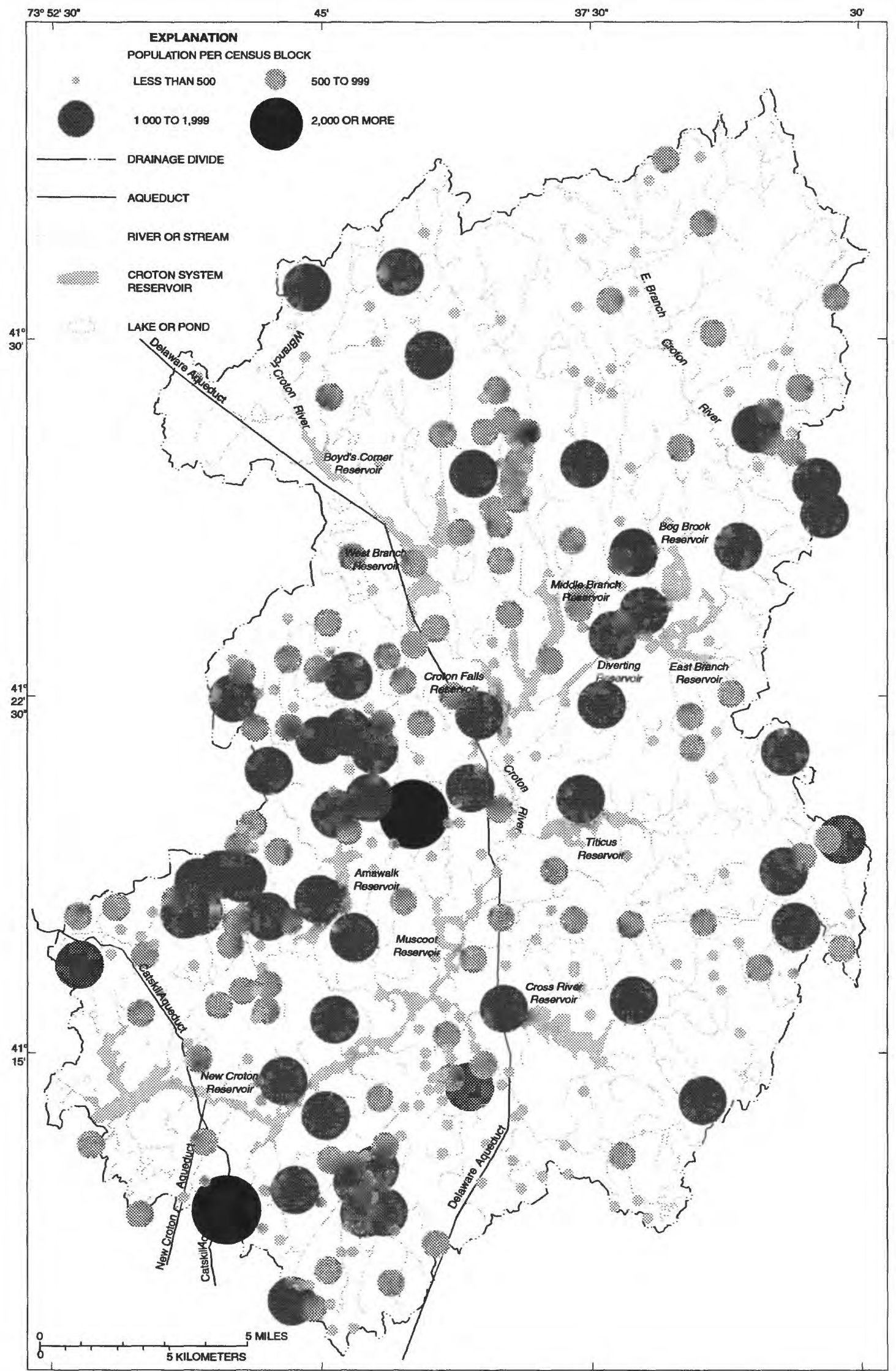

Base from U.S. Geological Survey digital data, 1:100,000, 1983

Figure 2. 1990 population within the Croton Reservoir system by census block. (Boundaries of census blocks not shown) (Data from U.S. Department of Comerce, 1990) 


\section{SITE SELECTION}

The process of identifying potential waterresources-monitoring sites began with the collection of water-resources information on past and present monitoring sites for water-quality sampling and other data such as population and land-use. The results of the data were organized and compiled into data sets, which were used to subjectively identify potential monitoring sites. The objective of final site selection was to adequately represent the water resources of the Croton Reservoir system with an optimum number of sites.

The following sections describe (1) the sources from which site-selection data were obtained, and (2) the process used to identify sites that are potentially suitable for the monitoring network.

\section{Data-Base Development}

Water-resources data from USGS and NYCDEP data bases were developed into GIS data sets and(or) compilations of data through the ARC/INFO ${ }^{1}$ GIS, and each GIS data set or data compilation was categorized into as one of four types-general information, surfacewater data, ground-water data, or water-quality data.

The GIS data base consists of a set of data "layers" or "coverages" that can be displayed as a map representing a specific category of data for a given geographic area. All data stored in a coverage are categorized as points, lines (arcs), or polygons. For example, well locations are represented as points, roads as arcs, and areas with a predominant land use as polygons. Coverages can be easily compared or overlain to identify intersections of spatial or point data, as was done to subjectively identify locations potentially suitable for monitoring sites. Additionally, the coverages can be combined to create maps that depict the contents (or partial contents) of the coverages. All maps in this report were created in this manner from the coverages developed in this study, but not all coverages developed for this study were used in production of these maps.

The following sections describe how the GIS coverages used to make the maps in this report were

${ }^{1}$ The use of trade or product names in this report is for identification purposes only and does not constitute endorsement by the U.S. Geological Survey. developed. Site names, locations, and types of data collected are listed in tables 1 through 7. Descriptions of all 13 GIS coverages and the two related data compilations are given in the appendix, which includes the type of coverage or data compilation, the map scale and the source of data, and the procedures used in development of each coverage or data array.

\section{General-Use Data}

The general-use data category refers to three data sets-hydrography, roads, and population.

Hydrography (fig. 1).-This data set represents the major surface-water features in the Croton Reservoir system-rivers, streams, aqueducts, reservoirs, and lakes. The major rivers are the Croton River, with its West, Middle, and East Branches, and the Muscoot River, the Titicus River, the Cross River, and the Kisco River (the four smaller tributaries to the Croton River). The 12 reservoirs in the study area, all of which supply water to New York City, are: Boyd's Corner (the smallest), West Branch, Middle Branch, East Branch, Bog Brook, Diverting, Croton Falls, Titicus, Cross River, Amawalk, Muscoot, and New Croton (the largest). The study area contains three aqueducts-the Catskill Aqueduct (which originates west of the study area, and although it has a connection at the New Croton Reservoir, its water usually bypasses the Croton watershed); the Delaware Aqueduct (which also originates west of the study area, and has connections to the northwest and southern ends of the West Branch Reservoir and to the Cross River Reservoir); and the New Croton Aqueduct (which originates within the study area at the New Croton Reservoir and is the primary source of water release for the Croton Basin). The hydrography coverage was developed from 1:100,000-scale digital line graphs (DLG) obtained from the USGS. It was then enhanced by digitizing smaller streams from 1:24,000-scale USGS topographic maps. A wetland area is represented by a line through the center of the wetland area rather than by the boundary of the wetland area.

Roads (fig. 1).-This coverage includes the transportation routes in the study area, from all major highways to most town roads and streets. The major highways in the study area are Interstate 84 in the northeastern part of the study area, the Taconic State Parkway in the western part, Interstate 684 (which intersects Interstate 84 in the northeast and runs south toward New York City), and the Sawmill River Parkway (which runs northeast to southwest and connects Interstate 684 with 
the Taconic State Parkway). This data set was developed from Digital Line Graphs (DLGs) (scale 1:100,000) obtained from the USGS. The linework from the DLGs was enhanced by including smaller roads from 1:24,000-scale planimetric maps provided by the New York State Department of Transportation.

Population (fig. 2).-This coverage represents the 1990 population distribution within the Croton Reservoir system. The study area had a population of 175,708 in 1990 (U.S. Department of Commerce, 1990). The largest concentrations are along the major transportation routes-south and east of the New Croton Reservoir (along Interstate 684 and the Sawmill River Parkway), around the Amawalk Reservoir (along the Taconic Parkway), and between the Middle Branch and Bog Brook Reservoirs (along Interstate 84). The population map (fig. 2) was derived from data of the 1990 Census of Population and Housing (U.S. Department of Commerce, 1990) for the conterminous United States. Each point in this map represents the approximate geographical center of a "census block group;" a census block group is the next to smallest geographic area in the Bureau of the Census hierarchy of geographic presentation. Data for each census block include the total number of persons and housing units.

\section{Surface-Water Data}

The surface-water data include four data sets-basins, dams, surface-water diversions (deliveries and withdrawals), and surface-water gages.

Basins (fig. 4).- This coverage includes the outline of the $374 \mathrm{mi}^{2}$ drainage basin and the boundaries of its 229 subbasins. The USGS drainage-area compilation for New York (Wagner, 1982) is continually updated, and, as part of this process, GIS coverages are being developed that contain the boundaries of all drainage basins and subbasins in New York. These boundaries are delineated by the USGS and checked by the Natural Resources Conservation Service.

Dams (Fig. 4).- This category contains information on dams on the streams and reservoirs in the Croton Reservoir system and includes the name of the owner of the dam and the body of water impounded by the dam (fig. 4). The information was obtained from a list of dams (July 1983) provided by NYSDEC from their Dam Safety Project data base. The study area contains 17 dams that are owned by New York City (these impound water in the 12 reservoirs in the study area) and 84 dams that are privately owned.
Deliveries and withdrawals.-This data compilation represents all deliveries and withdrawals from the Croton Reservoir system. The data, provided by the NYCDEP, included the losing or receiving body of water, the amount of water diverted, and the agency responsible for the diversion. Locations and amounts of 1990 deliveries and withdrawals are plotted in figure 3. The primary diversion ( $837.73 \mathrm{Mgal} / \mathrm{d}$ in 1990) into the Croton Reservoir system is from the Delaware Aqueduct into the northwestern end of the West Branch Reservoir (fig. 3); this water is generally diverted from the southern end of the West Branch Reservoir (909.78 $\mathrm{Mgal} / \mathrm{d}$ in 1990), where it is pumped into the next section of the Delaware Aqueduct. Diversions in the Croton Reservoir system include withdrawals by municipal and private water suppliers (ranging from less than $0.01 \mathrm{Mgal} / \mathrm{d}$ to $3.80 \mathrm{Mgal} / \mathrm{d}$ in 1990) and the diversion from the New Croton Reservoir to the New Croton Aqueduct (97.17 Mgal/d in 1990).

Surface-water gages (fig. 4, table 1).-This coverage includes information on past and present USGS surface-water data-collection sites. The USGS currently has one active streamflow-gaging station in the study area-Croton River at New Croton Dam near Croton-on-Hudson (site 01375000 , site 1 in fig. 4), which reported an annual average discharge of $680 \mathrm{ft}^{3} / \mathrm{s}$ for calendar year 1990. Historical streamflow data from two discontinued USGS gages were available-Croton River at Old Croton Dam near Croton Heights (site 01374990, site 3 in fig. 4), and Bird Brook near Croton (site 01375500 , site 2 in fig. 4). The USGS has also measured streamflow at 78 other locations (including low-flow sites, peak-flow sites, creststage gages, and miscellaneous-measurement sites) within the study area. The NYCDEP collects reservoir-stage data on each of the 12 reservoirs in the Croton Reservoir system.

Data on surface-water data-collection sites were obtained from USGS data bases and publications. Active and inactive USGS streamflow-measurement locations were obtained through a retrieval from the USGS National Water Information System (NWIS) data base and from USGS publications. The GIS data sets were then created from the location data obtained from these sources. Information in this data set includes the name of each site, USGS station number, type of data collected, drainage area, number of measurements made during the period of record, and the period of record. 


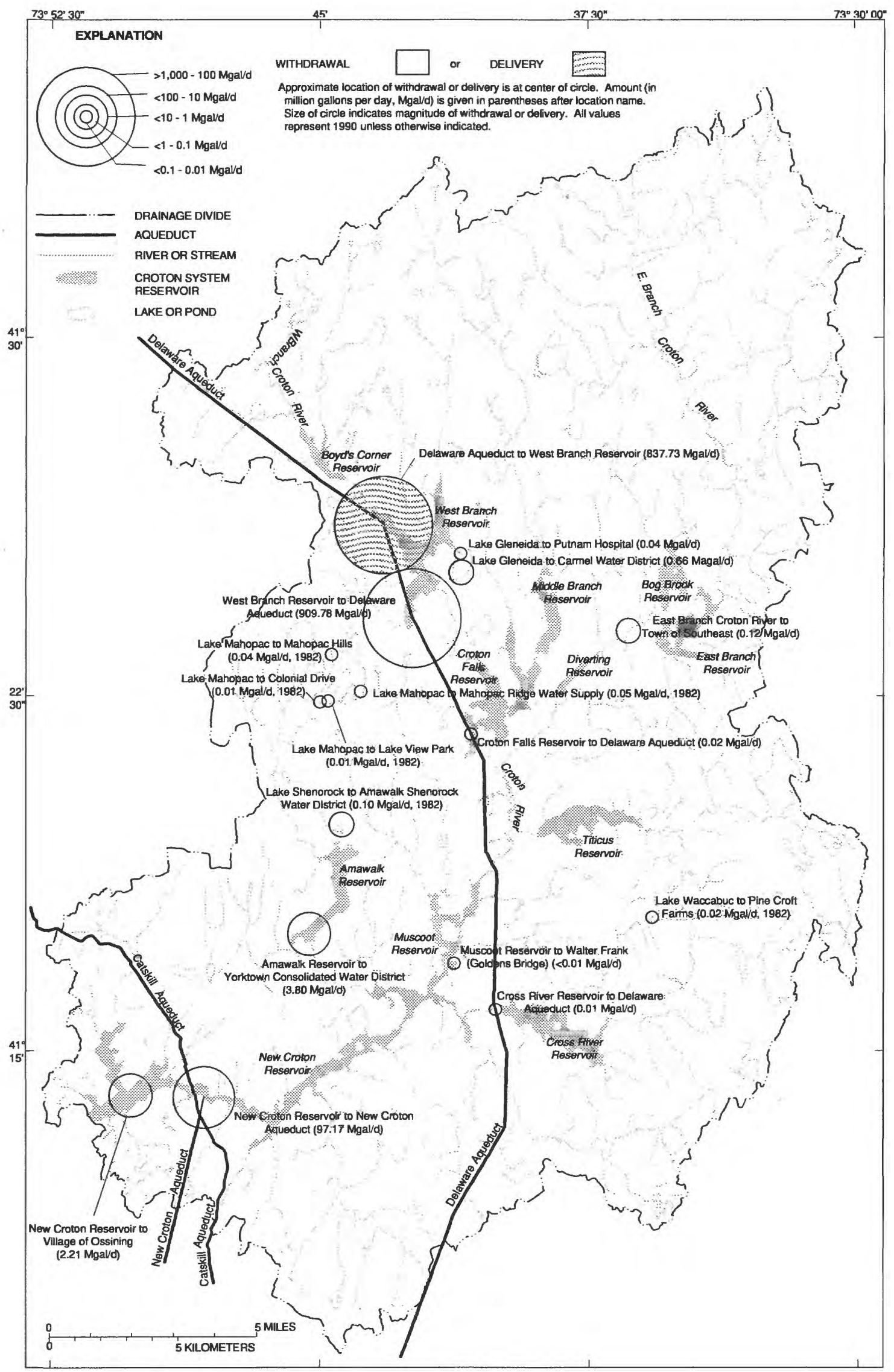

Base from U.S. Geological Survey digital data, 1:100,000, 1983

Figure 3. Annual 1990 withdrawal and delivery of surface water in the Croton Reservoir system 


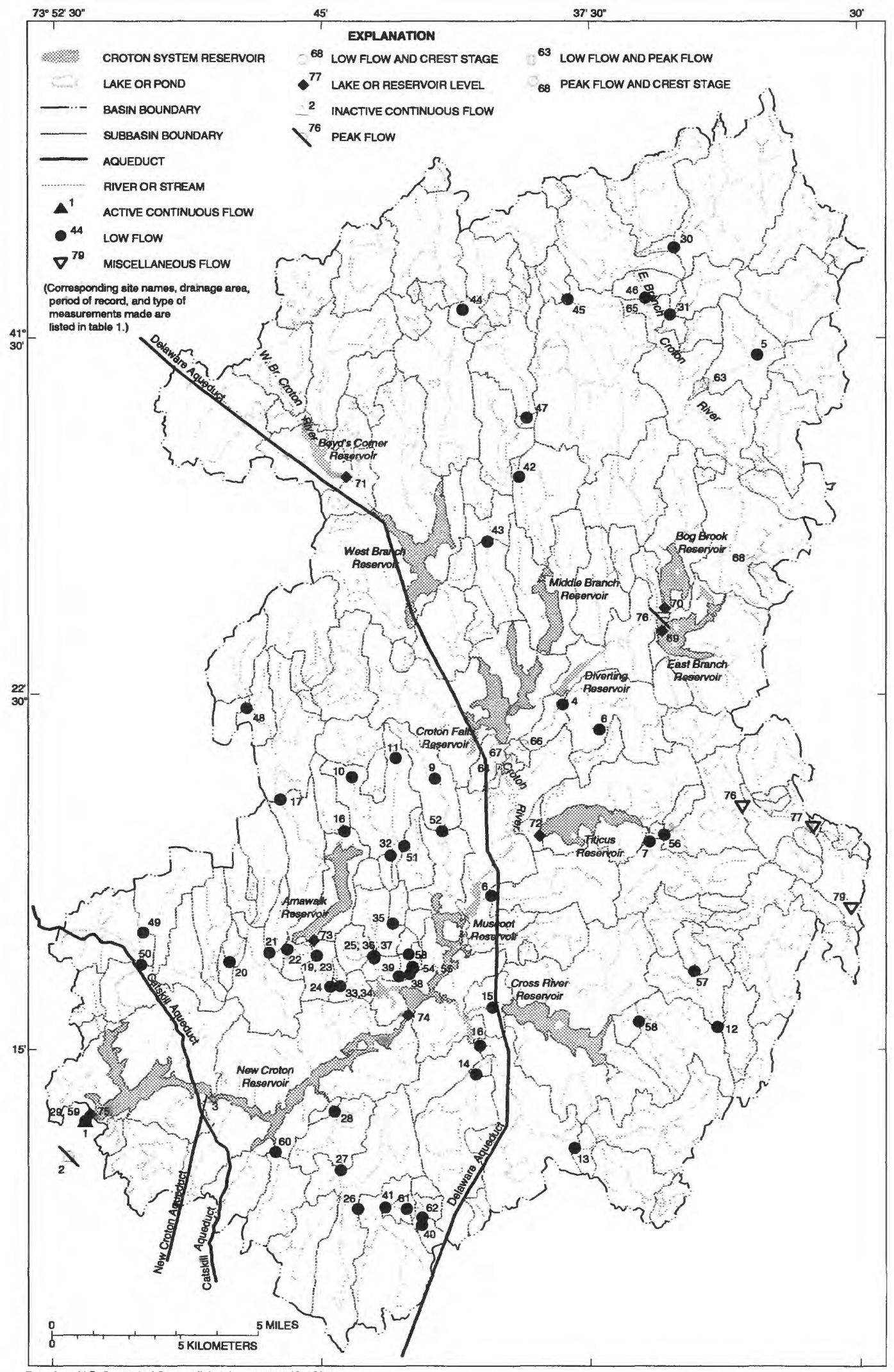

Base from U.S. Geological Survey digital data, 1:100,000, 1983

Figure 4. Drainage divides and surface-water flow, stage, or level sites established by the U.S. Geological Survey within the Croton Reservoir system 
Table 1. Types of data collected at surface-water data-collection sites.

[Locations are shown in fig. 4. - indicates data undetermined or not applicable; $\mathrm{LF}=$ low flow, ${ }^{*} \mathrm{PF}=$ peak flow, ${ }^{\dagger} \mathrm{CS}=$ crest stage, ${ }^{\ddagger} \mathrm{LK}=$ lake, $\mathrm{MS}=$ miscellaneous measurement, $\mathrm{ASF}=$ active continuous streamflow, $\mathrm{ISF}=$ inactive continuous streamflow.]

\begin{tabular}{|c|c|c|c|c|c|c|}
\hline $\begin{array}{l}\text { No. on } \\
\text { Fig. } 4\end{array}$ & $\begin{array}{c}\text { Site } \\
\text { identification } \\
\text { number }\end{array}$ & Site name & $\begin{array}{c}\text { Type of } \\
\text { mea- } \\
\text { sure- } \\
\text { ment }\end{array}$ & $\begin{array}{c}\text { Drain- } \\
\text { age } \\
\text { area }\end{array}$ & $\begin{array}{c}\text { No. of } \\
\text { meas. } \\
\text { for } \\
\text { period }\end{array}$ & Period of record \\
\hline 1 & 0137500000 & Croton River at New Croton Dam nr Croton-on-Hudson & ASF & 378 & 758 & $7 / 33$ to present \\
\hline 2 & 0137550000 & Bird Brook near Croton-on-Hudson & PF,ISF & 0.40 & - & - \\
\hline 3 & 0137499000 & Croton River at Old Croton Dam near Croton Heights & ISF & - & - & - \\
\hline 4 & 0137453500 & Holly Stream near Salem Center & LF & - & 1 & $08 / 15 / 74$ \\
\hline 5 & - & Haviland Hollow Brook near Haviland Hollow & LF & 10.10 & 2 & $03 / 25 / 54-09 / 29 / 54$ \\
\hline 6 & 0137454000 & Holly Stream near Brewster & LF & 4.82 & 8 & $07 / 10 / 62-09 / 28 / 73$ \\
\hline 7 & 0137478800 & Crook Brook at Salem Center & LF & 3.88 & 1 & $08 / 05 / 76$ \\
\hline 8 & 0137483000 & Croton River Tributary at Goldens Bridge & LF & 3.62 & 1 & $08 / 05 / 76$ \\
\hline 9 & - & Watermelon Creek near Lincolndale & LF & 1.06 & 1 & $03 / 24 / 54$ \\
\hline 10 & - & Plum Brook at Lake Lincolndale & LF & 1.67 & 2 & $03 / 24 / 54-09 / 29 / 54$ \\
\hline 11 & - & Plum Brook Tributary at Lake Lincolndale & LF & 0.27 & 2 & $03 / 24 / 54-09 / 29 / 54$ \\
\hline 12 & 0137488000 & Waccabuc River at Boutonville & LF & 10.62 & 2 & $07 / 07 / 76-08 / 04 / 76$ \\
\hline 13 & 0137490800 & Stone Hill River at Bedford & LF & 7.55 & 3 & $07 / 07 / 76-11 / 16 / 76$ \\
\hline 14 & 0137491500 & Broad Brook at Bedford Hills & LF & 5.11 & 1 & $08 / 15 / 74$ \\
\hline 15 & 0137491700 & Broad Brook at Katonah & LF & 5.30 & 1 & $08 / 05 / 76$ \\
\hline 16 & 0137491900 & Stone Hill River at Katonah & LF & 19.60 & 3 & $07 / 06 / 76-11 / 16 / 76$ \\
\hline 17 & 0137493000 & Muscoot River at Baldwin Place & LF & 13.50 & 5 & $03 / 24 / 54-11 / 16 / 76$ \\
\hline 18 & 0137493700 & Lake Shenorock Outlet at Shenorock & LF & 1.08 & 1 & $08 / 04 / 76$ \\
\hline 19 & 0137494200 & Muscoot River at Amawalk & LF & 19.90 & 3 & $07 / 06 / 76-07 / 21 / 76$ \\
\hline 20 & 0137494800 & Crum Pond Outlet at Yorktown Heights & LF & 3.88 & 1 & $08 / 04 / 76$ \\
\hline 21 & 0137496000 & Hallocks Mill Brook at Yorktown & LF & 10.40 & 3 & $07 / 06 / 76-07 / 21 / 76$ \\
\hline 22 & 0137496300 & Hallocks Mill Brook at Amawalk & LF & 11.40 & 2 & $07 / 19 / 76-07 / 21 / 76$ \\
\hline 23 & 0137496500 & Hallocks Mill Brook at Mouth at Amawalk & LF & 11.90 & 3 & $07 / 06 / 76-07 / 20 / 76$ \\
\hline 24 & 0137497000 & Muscoot River near Amawalk & LF & 32.60 & 3 & $07 / 06 / 76-07 / 21 / 76$ \\
\hline 25 & 0137497600 & Angle Fly Brook at Whitehall Corners & LF & 3.01 & 1 & $08 / 04 / 76$ \\
\hline 26 & 0137498300 & Kisco River at Lexington Ave. at Mount Kisco & LF & 6.06 & 4 & $08 / 16 / 74-11 / 16 / 76$ \\
\hline 27 & 0137498500 & Kisco River at Mount Kisco & LF & 一 & 1 & $08 / 16 / 74$ \\
\hline 28 & 0137498700 & Kisco River Below Mount Kisco & LF & 17.60 & 4 & $08 / 16 / 74-11 / 16 / 76$ \\
\hline 29 & - & Croton River Tributary near Croton-on-Hudson & LF & - & 1 & 03/18/63 \\
\hline 30 & 0137448800 & Brady Brook near Pawling & LF & 7.80 & 1 & $07 / 27 / 77$ \\
\hline 31 & 0137449100 & Stephens Brook near Patterson & LF & 1.45 & 1 & $07 / 27 / 77$ \\
\hline 32 & 0137485900 & Plum Brook Tributary at Lincolndale & LF & 0.25 & 2 & $10 / 06 / 87-11 / 16 / 87$ \\
\hline 33 & 0137497010 & Muscoot River Tributary near Whitehall Corners & LF & 0.16 & 2 & $10 / 09 / 87-11 / 16 / 87$ \\
\hline 34 & 0137497100 & Angle Fly Brook at Lincolndale & LF & 0.41 & 2 & $10 / 09 / 87-11 / 17 / 87$ \\
\hline 35 & 0137497200 & Angle Fly Brook Tributary 2 at Lincolndale & LF & 0.46 & 2 & 10/09/87-11/16/87 \\
\hline 36 & 0137497400 & Angle Fly Brook near Whitehall Corners & LF & 2.17 & 2 & $10 / 09 / 87-11 / 16 / 87$ \\
\hline 37 & 0137497500 & Angle Fly Brook Tributary 3 at Whitehall Corners & LF & 0.84 & 2 & 10/09/87-11/16/87 \\
\hline 38 & 0137497690 & Angle Fly Brook Trib. at Site 4 near Katonah & LF & 0.41 & 1 & $10 / 21 / 86$ \\
\hline 39 & 0137497700 & Angle Fly Brook Trib. at Site 5 near Katonah & LF & 0.56 & 3 & $10 / 21 / 86-11 / 16 / 87$ \\
\hline 40 & 0137498200 & Kisco River at Site 1 near Mount Kisco & LF & 0.35 & 1 & $10 / 22 / 86$ \\
\hline 41 & 0137498260 & Kisco River at Site 5 at Mount Kisco & LF & 1.42 & 1 & $10 / 22 / 86$ \\
\hline 42 & 0137465200 & Middle Branch Croton River at Lake Carmel & LF & 13.10 & - & - \\
\hline
\end{tabular}


Table 1. (continued) Types of data collected at surface-water data-collection sites.

\begin{tabular}{|c|c|c|c|c|c|c|}
\hline $\begin{array}{l}\text { No. on } \\
\text { Fig. } 4\end{array}$ & $\begin{array}{c}\text { Site } \\
\text { identification } \\
\text { number }\end{array}$ & Site name & $\begin{array}{c}\text { Type of } \\
\text { mea- } \\
\text { sure- } \\
\text { ment }\end{array}$ & $\begin{array}{l}\text { Drain- } \\
\text { age } \\
\text { area }\end{array}$ & $\begin{array}{l}\text { No. of } \\
\text { meas. } \\
\text { for } \\
\text { period }\end{array}$ & Period of record \\
\hline 43 & 0137467000 & Michael Brook at Carmel & LF & 1.72 & - & - \\
\hline 44 & 0137464000 & Stump Pond Stream at Ludingtonville & LF & 5.33 & 2 & $03 / 25 / 54-09 / 29 / 54$ \\
\hline 45 & - & Croton River Tributary, East Branch, at West Patterson & LF & 3.85 & 2 & $03 / 25 / 54-09 / 29 / 54$ \\
\hline 46 & 0137448900 & East Branch Croton River at Akins Corners & LF & 17.30 & 1 & $07 / 27 / 78$ \\
\hline 47 & 0137464500 & Stump Pond Stream at Kent Corners & LF & 10.30 & 17 & $02 / 21 / 75-08 / 19 / 80$ \\
\hline 48 & 0137492700 & Secor Brook at West Mahopac & LF & 2.65 & - & - \\
\hline 49 & 0137499200 & Hunter Brook near Yorktown & LF & 2.49 & 1 & $08 / 04 / 76$ \\
\hline 50 & 0137499300 & Hunter Brook Below Mill Pond near Yorktown & LF & 5.82 & 3 & $07 / 06 / 76-11 / 16 / 76$ \\
\hline 51 & 0137486000 & Plum Brook at Lincolndale & LF & 5.81 & 1 & 08/05/76 \\
\hline 52 & 0137484000 & Muscoot Reservoir Tributary at Somers & LF & 2.14 & 1 & $08 / 05 / 76$ \\
\hline 53 & 0137497650 & Angle Fly Brook Trib. at Site 1 near Katonah & LF & 0.20 & 3 & $10 / 21 / 86-11 / 16 / 87$ \\
\hline 54 & 0137497670 & Angle Fly Brook Trib. at Site 2 near Katonah & LF & 0.35 & 1 & $10 / 21 / 86$ \\
\hline 55 & 0137497680 & Angle Fly Brook Trib. at Site 3 near Katonah & LF & 0.38 & 1 & $10 / 21 / 86$ \\
\hline 56 & 0137478000 & Titicus River at Salem Center & LF & 12.40 & 4 & $08 / 15 / 74-11 / 16 / 76$ \\
\hline 57 & 0137487500 & Waccabuc River near South Salem & LF & - & - & - \\
\hline 58 & 0137489000 & Cross River near Cross River & LF & 17.10 & 4 & $08 / 15 / 74-11 / 16 / 76$ \\
\hline 59 & - & Croton River Tributary No. 2 near Croton-on-Hudson & LF & - & 1 & $03 / 18 / 63$ \\
\hline 60 & 0137498800 & Gedney Brook near Mount Kisco & LF & 2.01 & 1 & 08/04/76 \\
\hline 61 & 0137498250 & Kisco River at Site 4 near Mount Kisco & LF & 1.16 & 1 & 10/22/86 \\
\hline 62 & 0137498210 & Kisco River at Site 2 near Mount Kisco & LF & 0.38 & 1 & $10 / 22 / 86$ \\
\hline 63 & 0137449400 & Haviland Hollow Brook near Putnam Lake & LF,PF & 12.20 & 12 & $07 / 10 / 62-04 / 10 / 80$ \\
\hline 64 & 0137470500 & West Branch Croton River at Croton Falls & LF,PF & 54.30 & 1 & 08/05/76 \\
\hline 65 & 0137449000 & East Branch Croton River at Patterson & LF,PF & 17.60 & 2 & $03 / 25 / 54-09 / 29 / 54$ \\
\hline 66 & 0137454500 & East Branch Croton River at Croton Falls & LF,CS & 119.00 & 1 & 08/05/76 \\
\hline 67 & 0137454550 & East Branch Croton River at Croton Falls & LF,CS & - & 1 & $03 / 24 / 54$ \\
\hline 68 & 0137449490 & East Branch Croton River Trib. near Deforest Corners & $\mathrm{PF}, \mathrm{CS}$ & 0.61 & 3 & $01 / 09 / 78-04 / 10 / 80$ \\
\hline 69 & 0137449500 & East Branch Croton Reservoir at Sodom & LK & - & - & - \\
\hline 70 & 0137450000 & Bog Brook Reservoir at Sodom & LK & - & - & - \\
\hline 71 & 0137458000 & Boyd Corners Reservoir near Clear Pool Camp & LK & - & - & - \\
\hline 72 & 0137482000 & Titicus Reservoir at Purdy Station & LK & - & - & - \\
\hline 73 & 0137494000 & Muscoot River (Amawalk Res) at Dam at Amawalk & LK & - & - & - \\
\hline 74 & 0137498000 & Croton River at Muscoot Reservoir Dam at Katonah & LK & - & - & - \\
\hline 75 & 0137490500 & New Croton Reservoir near Croton-on-Hudson & LK & - & - & - \\
\hline 76 & 0137449600 & East Branch Croton River near Brewster & PF & 79.50 & - & - \\
\hline 77 & - & Titicus R. Below Lake Naraneka & MS & - & - & - \\
\hline 78 & - & Titicus River nr North Salem & MS & - & - & - \\
\hline 79 & - & Titicus River nr Ridgefield & MS & - & - & - \\
\hline
\end{tabular}

*Low flow, LF, is a site where instantaneous discharge measurements have been made during a low flow period.

${ }^{\dagger}$ Peak flow, PF, is a site where instantaneous or continuous discharges have been measured or determined during a peak flow period.

${ }^{\ddagger}$ Crest stage, CS, is a site where peak stage is measured during a peak flow period and may or may not have a calculated discharge associated with the peak stage. 


\section{Ground-Water Data}

Four data sets were developed for the groundwater data category - surficial geology (fig. 5A), bedrock geology (fig. 5B), selected potentially high-yielding wells, and ground-water withdrawal sites for public water supply.

Surficial and bedrock geology (figs. 5A, 5B).- These data sets represent the boundaries of the surficial deposits and the bedrock formations; the boundary data were digitized from bedrock and surficial geology maps obtained from the New York State Geological Survey (Cadwell and others, 1986; Fisher and others, 1970). The surficial material in the study area consists primarily of till. Recent deposits and(or) stratified drift are present in many streams valleys, but only the most extensive deposits are shown in figure 5A. Recent deposits include swamp deposits or alluvium and outwash sand and gravel. Most wells finished in stratified drift are generally more productive than wells finished in other surficial withdrawals. The five most common types of bedrock in the study area are the Biotite-quartz-plagioclasegneiss (bqpc), the Inwood marble (OEi), the Manhattan schist (Om), the Fordham gneiss (f), and the Amphibolite (am) units.

Potential sites for high-yielding wells (figs. 5A, 5B).- The ground-water data coverage (figures 5A and $5 \mathrm{~B}$ ) represents ground-water withdrawal sites within the study area. Figure $5 \mathrm{~A}$ represents 30 wells screened in surficial aquifers, and figure 5B represents 102 wells finished in bedrock aquifers. The largest yield from a surficial aquifer (\#93, Westchester 1063, near Cross River Reservoir) was $600 \mathrm{gal} /$ $\mathrm{min}$, and the largest yield for a bedrock well (\#24, Westchester 2048 near Croton Falls Reservoir) was $235 \mathrm{gal} / \mathrm{min}$. The data base includes the rate of water withdrawn at each site, site-identification numbers, and source aquifers (table 2). These data were obtained from the USGS NWIS data base and represents all wells in the study area that had a reported yield of at least $50 \mathrm{gal} / \mathrm{min}$.

Ground-water-withdrawal sites for public water supply (figs. 5A, 5B).- - This data base includes the amount of ground water withdrawn at each site, the population served, and well-identification numbers (table 2). A retrieval of public-water-supply groundwater withdrawal sites was made from the USGS NWIS data base; the data for this data set originally came from the New York State Department of Health and represent the year 1981. The largest amount of water withdrawn from a ground-water source by a public water supplier in the study area was $459 \mathrm{gal} / \mathrm{min}$ by Bedford Consolidated (\#61); the smallest amount withdrawn was $0.69 \mathrm{gal} / \mathrm{min}$ by Spring Knoll Estates (\#5).

\section{Water-Quality Data}

The water-quality data category contains two data sets-water-quality data-collection sites and land use; also included is a data compilation listing sites that may be point sources of pollution.

Water-quality data collection sites.-This coverage contains information on the locations of past and present USGS and NYCDEP water-quality sites within the study area (fig. 6) and includes the identification number, site name, type of site, period of record, and which chemical analyses were completed on samples from each site. These data are listed in table 3 . The USGS had made water-quality measurements at 42 surface-water sites, 53 ground-water sites, and 19 lake or reservoir sites in this study area before this study began (1990). The NYCDEP currently collects water-quality data at 45 sites on reservoirs, 23 sites on streams or rivers, and at 1 aqueduct.

Data on water-quality data collection sites were obtained from USGS NWIS databases and from the NYCDEP. A retrieval was made from the USGS NWIS Water-Quality Database to identify all USGS data-collection sites (ground water or surface water) in the study area at which water samples had been previously collected for analysis; information on these sites included the site name, the type of site (ground water, surface water, or lake/reservoir), the latitude and longitude, number of samples collected and analyzed, the range of dates of sample collection (samples were collected as early as 1964), and the physical, chemical, and biological constituents for which each sample was analyzed. Also included in the water-quality data-collection site coverage are the locations of surface-water sites run by the NYCDEP at which water samples have been collected for chemical analysis. The NYCDEP provided maps showing locations of the sampling sites and tables containing the following information on each site: type of site (reservoir, stream, aqueduct), number of samples collected, frequency of sample collection, period(s) during which samples were collected, and the physical, chemical, and biological constituents for which the samples were analyzed.

Land use.-The land-use coverage represents the major land-use practices in the study area. Land- 


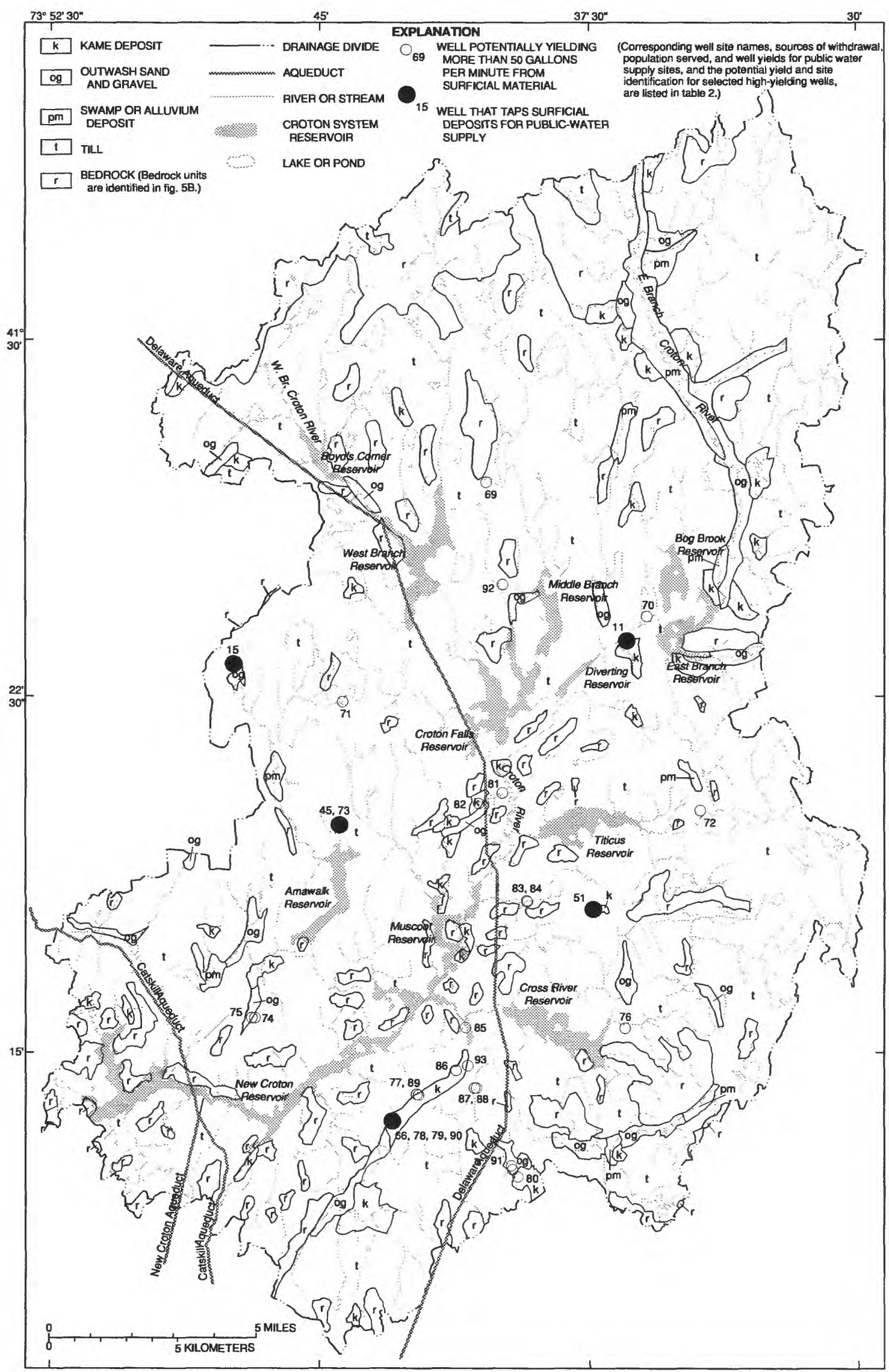

Base from U.S. Geological Survey digital data, 1:100,000, 1983

Figure 5A. Surficial geology and location of public-supply wells and wells yielding at least 50 gallons per minute from surficial deposits within the Croton Reservoir system. 


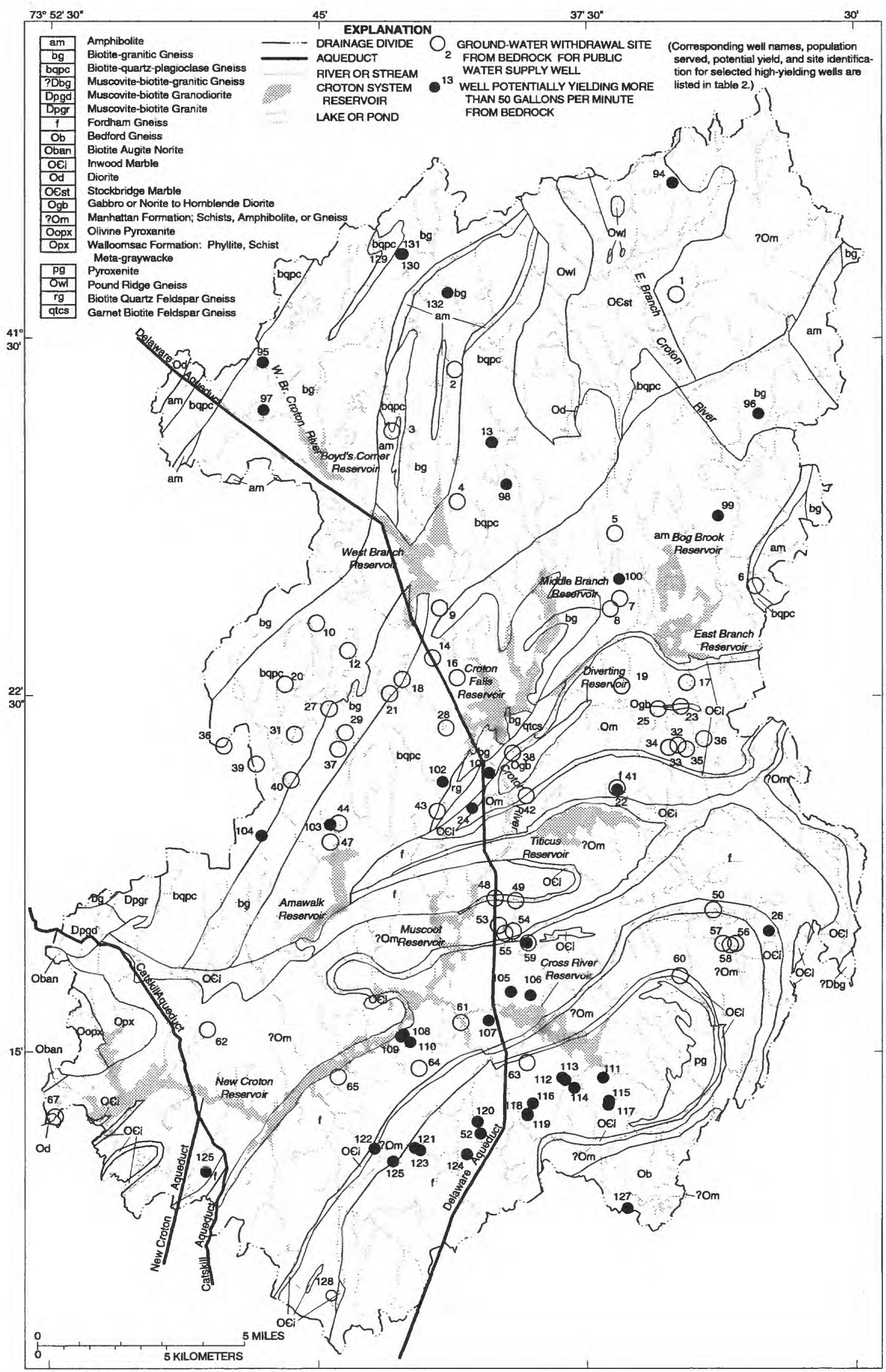

Base from U.S. Geological Survey digital data, 1:100,000, 1983

Figure 5B. Bedrock geology and location of public-supply wells finished in bedrock and wells yielding at least 50 gallons per minute from bedrock within the Croton Reservoir system. 
Table 2. Data on ground-water withdrawal sites for public water supply and selected high-yielding wells [Well locations are shown in figs. 5A and 5B. Dash indicates not applicable.]

\begin{tabular}{|c|c|c|c|c|c|c|}
\hline $\begin{array}{l}\text { Well no. } \\
\text { in figs. } \\
5 A \text { or } 5 B\end{array}$ & $\begin{array}{c}\text { Site-identification } \\
\text { number }\end{array}$ & Site name & $\begin{array}{l}\text { Aquifer } \\
\text { (B, bed- } \\
\text { rock; } \mathrm{S} \text {, } \\
\text { surficial } \\
\text { deposit) }\end{array}$ & $\begin{array}{l}\text { Popula- } \\
\text { tion } \\
\text { served }\end{array}$ & $\begin{array}{l}\text { Reported rate of } \\
\text { ground-water } \\
\text { withdrawal } \\
\text { (gal/min) }\end{array}$ & $\begin{array}{l}\text { Potential rate of } \\
\text { ground-water } \\
\text { withdrawal } \\
\text { (gal/min) }\end{array}$ \\
\hline 1 & 413055073345901 & Alpine Village & $\mathbf{B}$ & 340 & 17 & - \\
\hline 2 & 412920073411301 & Leeside Estates & B & 256 & 18 & - \\
\hline 3 & 412803073425901 & Gypsy Trail Club & B & 300 & 14 & - \\
\hline 4 & 412634073410901 & Kent Wd. No. 1 & B & 360 & 26 & - \\
\hline 5 & 412554073364301 & Spring Knoll Estates & B & 20 & 0.69 & - \\
\hline 6 & 412447073324901 & Wildwood Homes & B & 148 & 2.8 & - \\
\hline 7 & 412431073363501 & Blackberry Hill & B & 400 & 28 & - \\
\hline 8 & 412418073365101 & First Brewster Corp. & B & 255 & 31 & - \\
\hline 9 & 412419073413901 & West Branch Acres & B & 240 & 20 & - \\
\hline 10 & 412400073450501 & London Bridge Water Works & B & 288 & 14 & - \\
\hline 11 & 412340073362701 & Brewster Village & $S$ & 3,200 & 210 & - \\
\hline 12 & 412326073441301 & Mahopac Lake Shore Estates & B & 80 & 6.1 & - \\
\hline 13 & 412749073401101 & Putnam 1083 & B & - & - & 150 \\
\hline 14 & 412317073415101 & Carmel Wd. No. 7-Tomahawk C & B & 68 & 5.6 & - \\
\hline 15 & 412311073472401 & Crescent Road Water Supply & $\mathbf{S}$ & 20 & 1.7 & - \\
\hline 16 & 412252073410901 & Country Hill Estates & B & 200 & 14 & - \\
\hline 17 & 412246073344301 & Fox Hill Estates & B & 128 & 2.6 & - \\
\hline 18 & 412250073424201 & Carmel Wd. No 6-Shell Valy & B & 324 & 18 & - \\
\hline 19 & 412242073363201 & George Walsh & B & 48 & 3.3 & - \\
\hline 20 & 412244073455801 & Red Mills Water Supply & B & 400 & 28 & - \\
\hline 21 & 412232073430301 & Capri Estates & B & 140 & 9.7 & - \\
\hline 22 & 412038073364101 & Westchester 461 & B & - & 100 & - \\
\hline 23 & 412216073345301 & Vails Grove & B & 510 & 16 & - \\
\hline 24 & 412007073404401 & Westchester 2048 & B & - & 235 & - \\
\hline 25 & 412213073353101 & Star Ridge Manor & B & 368 & 3.5 & - \\
\hline 26 & 411733073322701 & R 4 & B & - & 220 & - \\
\hline 27 & 412213073444401 & Indian Hill & B & 56 & 2.6 & - \\
\hline 28 & 412149073412801 & Union Valley Estates & B & 290 & 20 & - \\
\hline 29 & 412143073441701 & Mahopac Water Co. & B & 500 & 42 & - \\
\hline 30 & 412135073341501 & Pietschs Gardens & B & 250 & 8.3 & - \\
\hline 31 & 412141073454201 & Carmel Wd. No. 5-maple Terr & B & 180 & 12 & - \\
\hline 32 & 412127073345801 & Bloomerside Realty Inc. & B & 100 & 3.5 & 一 \\
\hline 33 & 412129073350301 & Bloomerside Realty Inc. & B & 100 & 3.5 & - \\
\hline 34 & 412124073351301 & Bloomerside Realty Inc. & B & 100 & 3.5 & - \\
\hline 35 & 412122073344401 & Pabst Water Co. Inc. & B & 260 & 6.7 & - \\
\hline 36 & 412126073474001 & Wood Hill Estates & B & 100 & 4.2 & - \\
\hline 37 & 412122073442901 & Chateau Ridge & B & 300 & 21 & - \\
\hline 38 & 412117073393701 & Juengstville Farm Assoc. & B & 50 & 3.5 & - \\
\hline 39 & 412103073464601 & Boniville Water Co. & B & 360 & 27 & - \\
\hline 40 & 412043073454801 & Carmel Wd. No. 4-Baldwin WC & B & 1,600 & 17 & - \\
\hline 41 & 412033073364001 & Salem Acres Association & B & 154 & 10 & - \\
\hline 42 & 412023073391401 & Sunset Ridge Wd. & B & 600 & 28 & - \\
\hline
\end{tabular}


Table 2. (Continued) Data on ground-water withdrawal sites for public water supply and selected high-yielding wells

\begin{tabular}{|c|c|c|c|c|c|c|}
\hline $\begin{array}{l}\text { Well no. } \\
\text { in figs. } \\
5 \mathrm{~A} \text { or } 5 \mathrm{~B}\end{array}$ & $\begin{array}{c}\text { Site-identification } \\
\text { number }\end{array}$ & Site name & $\begin{array}{l}\text { Aquifer } \\
\text { (B, bed- } \\
\text { rock; } \mathrm{S} \text {, } \\
\text { surficial } \\
\text { deposit) }\end{array}$ & $\begin{array}{l}\text { Popula- } \\
\text { tion } \\
\text { served }\end{array}$ & $\begin{array}{l}\text { Reported rate of } \\
\text { ground-water } \\
\text { withdrawal } \\
\text { (gal } / \mathrm{min})\end{array}$ & $\begin{array}{l}\text { Potential rate of } \\
\text { ground-water } \\
\text { withdrawal } \\
\text { (gal } / \mathrm{min})\end{array}$ \\
\hline 43 & 412003073414301 & Greenbriar Subdivision & $\bar{B}$ & 240 & 17 & - \\
\hline 44 & 411948073442801 & Amawalk-Shenorock Wd. & B & 480 & 28 & - \\
\hline 45 & 411948073442701 & Amawalk-Shenorock Wd. & $S$ & 480 & 28 & - \\
\hline 46 & 411718073391301 & Westchester 2023 & B & - & - & 100 \\
\hline 47 & 411924073444201 & Horton Estates Water Trust & B & 200 & 8.3 & - \\
\hline 48 & 411814073400601 & Candlewood Park & B & 175 & 9.7 & - \\
\hline 49 & 411811073393201 & Wild Oaks Water Co. & B & 410 & 21 & - \\
\hline 50 & 411759073340001 & Twin Lakes WW Corp. & B & 350 & 17 & - \\
\hline 51 & 411801073372401 & Indian Hill Subdivision & $\mathbf{S}$ & 96 & 6.7 & - \\
\hline 52 & 411325073403201 & Westchester 449 & B & - & - & 100 \\
\hline 53 & 411740073400001 & Golden Bridge Community & B & 43 & 3.0 & - \\
\hline 54 & 411733073393601 & Golden Bridge Community & B & 43 & 3.0 & - \\
\hline 55 & 411730073395001 & Golden Bridge Community & B & 44 & 3.0 & - \\
\hline 56 & 411717073332301 & Truesdale Lake Prop. Owner & B & 133 & 6.0 & - \\
\hline 57 & 411717073334401 & Truesdale Lake Prop. Owner & B & 133 & 6.0 & - \\
\hline 58 & 411715073333201 & Truesdale Lake Prop. Owner & B & 134 & 6.0 & - \\
\hline 59 & 411718073391101 & Lake Katonah Club Inc. & B & 390 & 20 & - \\
\hline 60 & 411636073345501 & Pamela Lane Water Supply & B & 40 & 2.4 & - \\
\hline 61 & 411537073410301 & Bedford Consolidated & B & 6,150 & 460 & - \\
\hline 62 & 411528073480701 & Westview Well Assoc & B & 18 & 1.2 & - \\
\hline 63 & 411446073391301 & Bedford Farms Water Co. & B & 280 & 5.6 & 一 \\
\hline 64 & 411439073421401 & Roosevelt Drive Water Users & B & 84 & 6.5 & - \\
\hline 65 & 411428073442901 & Cedar Downs Wd. & B & 251 & 8.9 & - \\
\hline 66 & 411334073425901 & Mount Kisco Village & $S$ & 9,000 & 200 & - \\
\hline 67 & 411338073522201 & Windsor Oaks Prop. Owners & B & 55 & 3.8 & - \\
\hline 68 & 412054073393701 & Croton Falls Wd. & B & 250 & 8.5 & - \\
\hline 69 & 412659073402601 & Putnam 154 & $S$ & - & - & 50 \\
\hline 70 & 412410073360101 & Putnam 825 & $S$ & - & - & 70 \\
\hline 71 & 412222073442101 & Putnam 566 & $S$ & - & - & 50 \\
\hline 72 & 412018073343201 & Westchester 464 & $S$ & - & - & 80 \\
\hline 73 & 411947073442801 & Westchester 1460 & $S$ & - & - & 50 \\
\hline 74 & 411546073465101 & Westchester 549 & $S$ & - & - & 50 \\
\hline 75 & 411547073465701 & Westchester 548 & $S$ & - & - & 50 \\
\hline 76 & 411535073363301 & Westchester 322 & $S$ & 一 & - & 70 \\
\hline 77 & 411408073421901 & Westchester 2001 & $S$ & - & - & 60 \\
\hline 78 & 411342073430501 & Westchester 505 & $S$ & - & - & 70 \\
\hline 79 & 411341073430201 & Westchester 504 & $S$ & - & - & 70 \\
\hline 80 & 411228073372301 & Westchester 695 & $S$ & - & - & 50 \\
\hline 81 & 412028073395601 & Westchester 2049 & $S$ & - & - & 350 \\
\hline 82 & 412015073403601 & Westchester 2047 & $\mathbf{S}$ & - & - & 146 \\
\hline 83 & 411812073391501 & Westchester 2028 & $S$ & - & - & 120 \\
\hline 84 & 411811073391401 & Westchester 2029 & $S$ & - & - & 120 \\
\hline 85 & 411532073405801 & Westchester 501 & $S$ & - & - & 400 \\
\hline 86 & 411440073411401 & Westchester 502 & $\mathbf{S}$ & - & - & 180 \\
\hline 87 & 411415073403701 & Westchester 991 & $\mathbf{S}$ & - & - & 300 \\
\hline
\end{tabular}


Table 2. (Continued) Data on ground-water withdrawal sites for public water supply and selected high-yielding wells

\begin{tabular}{|c|c|c|c|c|c|c|}
\hline $\begin{array}{l}\text { Well no. } \\
\text { in figs. } \\
5 \mathrm{~A} \text { or } 5 \mathrm{~B}\end{array}$ & $\begin{array}{c}\text { Site-identification } \\
\text { number }\end{array}$ & Site name & $\begin{array}{l}\text { Aquifer } \\
\text { (B, bed- } \\
\text { rock; } S \text {, } \\
\text { surficial } \\
\text { deposit) }\end{array}$ & $\begin{array}{l}\text { Popula- } \\
\text { tion } \\
\text { served }\end{array}$ & $\begin{array}{l}\text { Reported rate of } \\
\text { ground-water } \\
\text { withdrawal } \\
\text { (gal/min) }\end{array}$ & $\begin{array}{l}\text { Potential rate of } \\
\text { ground-water } \\
\text { withdrawal } \\
\text { (gal/min) }\end{array}$ \\
\hline 88 & 411416073404301 & Westchester 2022 & $\mathbf{S}$ & - & - & 270 \\
\hline 89 & 411407073421601 & Westchester 2002 & $\mathbf{S}$ & 一 & - & 120 \\
\hline 90 & 411335073430401 & Westchester 503 & $\mathbf{S}$ & - & - & 150 \\
\hline 91 & 411232073393901 & Westchester 4824 & S & - & - & 120 \\
\hline 92 & 412451073395501 & Putnam 787 & $\mathbf{S}$ & - & - & 500 \\
\hline 93 & 411448073405201 & Westchester 1063 & S & - & - & 600 \\
\hline 94 & 413316073350501 & Dutchess 1804 & B & - & - & 60 \\
\hline 95 & 412928073463201 & Putnam 586 & B & - & - & 50 \\
\hline 96 & 412825073324301 & Putnam 1104 & B & - & - & 50 \\
\hline 97 & 412830073463401 & Putnam 1082 & B & - & - & 50 \\
\hline 98 & 412656073394701 & Putnam 1043 & B & - & - & 50 \\
\hline 99 & 412616073335101 & Putnam 1139 & B & - & - & 80 \\
\hline 100 & 412456073363601 & Putnam 1151 & B & 一 & - & 50 \\
\hline 101 & 412052073401601 & Westchester 1231 & B & - & - & 60 \\
\hline 102 & 412041073413401 & Westchester 2031 & B & 一 & - & 66 \\
\hline 103 & 411946073444201 & Westchester 1434 & B & - & 一 & 50 \\
\hline 104 & 411932073463801 & Westchester 539 & B & - & - & 75 \\
\hline 105 & 411616073394001 & Westchester 1319 & B & - & - & 50 \\
\hline 106 & 411612073391001 & Westchester 4271 & B & $一$ & 一 & 50 \\
\hline 107 & 411540073401701 & Westchester 4268 & B & 一 & - & 60 \\
\hline 108 & 411522073424001 & Westchester 4437 & B & - & - & 75 \\
\hline 109 & 411519073424401 & Westchester 4912 & B & - & - & 75 \\
\hline 110 & 411512073422901 & Westchester 4326 & B & - & - & 50 \\
\hline 111 & 411428073371101 & Westchester 4198 & B & - & - & 50 \\
\hline 112 & 411428073381601 & Westchester 4307 & B & - & - & 55 \\
\hline 113 & 411432073381201 & Westchester 1250 & B & - & - & 60 \\
\hline 114 & 411414073375601 & Westchester 4390 & B & - & - & 50 \\
\hline 115 & 411357073365701 & Westchester 4443 & B & - & - & 60 \\
\hline 116 & 411402073390401 & Westchester 456 & B & - & - & 65 \\
\hline 117 & 411353073365501 & Westchester 4866 & B & - & - & 60 \\
\hline 118 & 411342073391201 & Westchester 4870 & B & - & 一 & 60 \\
\hline 119 & 411340073391201 & Westchester 4447 & B & - & 一 & 60 \\
\hline 120 & 411337073404201 & Westchester 451 & B & - & - & 60 \\
\hline 121 & 411259073422101 & Westchester 4310 & B & - & - & 75 \\
\hline 122 & 411303073432901 & Westchester 1058 & B & - & - & 50 \\
\hline 123 & 411301073421501 & Westchester 455 & B & - & - & 50 \\
\hline 124 & 411251073405301 & Westchester 4894 & B & - & - & 50 \\
\hline 125 & 411242073425701 & Westchester 4169 & B & 一 & - & 50 \\
\hline 126 & 411229073480001 & Westchester 931 & B & - & - & 50 \\
\hline 127 & 411145073362501 & Westchester 4325 & B & 一 & 一 & 50 \\
\hline 128 & 410954073443101 & Westchester 976 & B & - & 一 & 75 \\
\hline 129 & 413146073424101 & Dutchess 1659 & B & - & - & 109 \\
\hline 130 & 413146073424102 & Dutchess 1089 & B & - & - & 109 \\
\hline 131 & 413146073424401 & Dutchess 1658 & B & - & - & 103 \\
\hline 132 & 413057073412501 & Dutchess 1774 & B & - & - & 100 \\
\hline
\end{tabular}




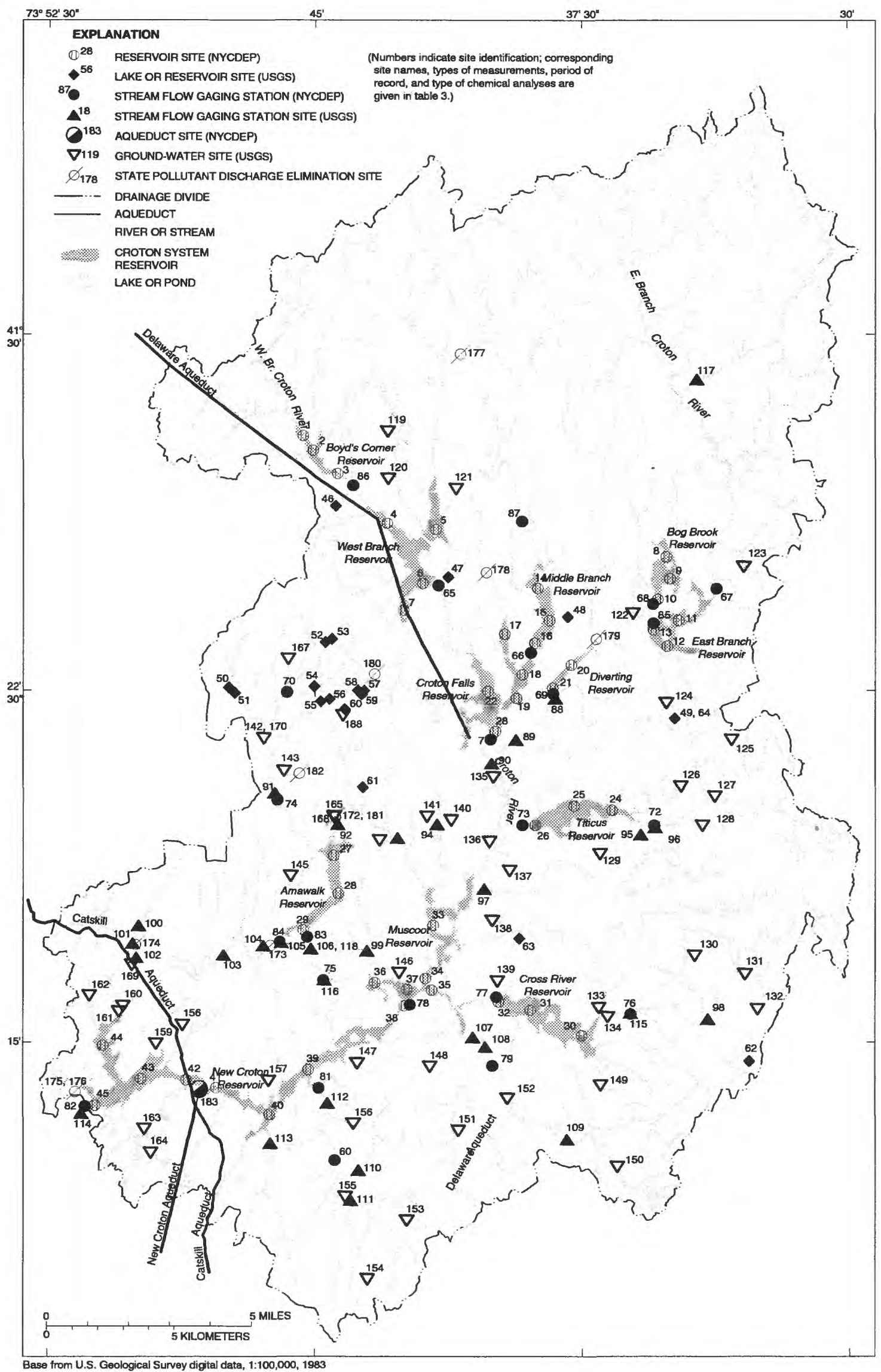

Figure 6. Past and current U.S. Geological Survey and New York City Department of Environmental Protection water-quality-data collection sites in the Croton Reservoir system. 
Table 3. Summary of data collected at water-quality sites

[-, no USGS site-identification number assigned; n/a, no data available; LK-RES, lake or reservoir site; SW, surface-water site; GW, ground-water site; KEY POINT, aqueduct site; B, background water-quality constituents; N, nitrogen; P, phosphorus; $\mathrm{Q}$, discharge; $\mathrm{O}$, other constituents.]

\begin{tabular}{|c|c|c|c|c|c|}
\hline $\begin{array}{l}\text { No. on } \\
\text { fig. } 6\end{array}$ & $\begin{array}{c}\text { Site- } \\
\text { identification } \\
\text { number }\end{array}$ & Site name & $\begin{array}{c}\text { Type of } \\
\text { site }\end{array}$ & $\begin{array}{c}\text { Period of } \\
\text { Record }\end{array}$ & $\begin{array}{c}\text { Constituents } \\
\text { analyzed }\end{array}$ \\
\hline 1 & - & Boyd Corners 3 & LK-RES & 1991-present & BONP \\
\hline 2 & - & Boyd Corners 2 & LK-RES & 1991-present & BONP \\
\hline 3 & - & Boyd Corners 1 & LK-RES & 1991-present & BONP \\
\hline 4 & - & West Branch 3 & LK-RES & 1984-present & BONP \\
\hline 5 & - & West Branch 4 & LK-RES & 1984-present & BONP \\
\hline 6 & - & West Branch 2 & LK-RES & 1984-present & BONP \\
\hline 7 & - & West Branch 1 & LK-RES & 1984-present & BONP \\
\hline 8 & - & Bog Brook 3 & LK-RES & 1986-present & BONP \\
\hline 9 & - & Bog Brook 2 & LK-RES & 1986-present & BONP \\
\hline 10 & - & Bog Brook 1 & LK-RES & 1986-present & BONP \\
\hline 11 & - & East Branch 3 & LK-RES & 1986-present & BONP \\
\hline 12 & - & East Branch 2 & LK-RES & 1986-present & BONP \\
\hline 13 & - & East Branch 1 & LK-RES & 1986-present & BONP \\
\hline 14 & - & Middle Branch 3 & LK-RES & 1986-present & BONP \\
\hline 15 & - & Middle Branch 2 & LK-RES & 1986-present & BONP \\
\hline 16 & - & Middle Branch 1 & LK-RES & 1986-present & BONP \\
\hline 17 & - & Croton Falls 5 & LK-RES & 1986-present & BONP \\
\hline 18 & - & Croton Falls 4 & LK-RES & 1986-present & BONP \\
\hline 19 & - & Croton Falls 3 & LK-RES & 1986-present & BONP \\
\hline 20 & - & Diverting 2 & LK-RES & 1986-present & BONP \\
\hline 21 & - & Diverting 1 & LK-RES & 1986-present & BONP \\
\hline 22 & - & Croton Falls 2 & LK-RES & 1986-present & BONP \\
\hline 23 & - & Croton Falls 1 & LK-RES & 1986-present & BONP \\
\hline 24 & - & Titicus 3 & LK-RES & 1986-present & BONP \\
\hline 25 & - & Titicus 2 & LK-RES & 1986-present & BONP \\
\hline 26 & - & Titicus 1 & LK-RES & 1986-present & BONP \\
\hline 27 & - & Amawalk 3 & LK-RES & 1986-present & BONP \\
\hline 28 & - & Amawalk 2 & LK-RES & 1986-present & BONP \\
\hline 29 & - & Amawalk 1 & LK-RES & 1986-present & BONP \\
\hline 30 & - & Cross River 3 & LK-RES & 1986-present & BONP \\
\hline 31 & - & Cross River 2 & LK-RES & 1986-present & BONP \\
\hline 32 & - & Cross River 1 & LK-RES & 1986-present & BONP \\
\hline 33 & - & Muscoot 6 & LK-RES & 1984-present & BONP \\
\hline 34 & - & Muscoot 5 & LK-RES & 1984-present & BONP \\
\hline 35 & - & Muscoot 4 & LK-RES & 1984-present & BONP \\
\hline 36 & - & Muscoot 2 & LK-RES & 1984-present & BONP \\
\hline 37 & - & Muscoot 3 & LK-RES & 1984-present & BONP \\
\hline 38 & - & Muscoot 1 & LK-RES & 1984-present & BONP \\
\hline 39 & - & Croton 7 & LK-RES & 1984-present & BONP \\
\hline 40 & - & Croton 6 & LK-RES & 1984-present & BONP \\
\hline 41 & - & Croton 5 & LK-RES & 1984-present & BONP \\
\hline
\end{tabular}


Table 3. (Continued) Summary of data collected at water-quality sites

\begin{tabular}{|c|c|c|c|c|c|}
\hline $\begin{array}{l}\text { No. on } \\
\text { fig. } 6\end{array}$ & $\begin{array}{c}\text { Site- } \\
\text { identification } \\
\text { number }\end{array}$ & Site name & $\begin{array}{l}\text { Type of } \\
\text { site }\end{array}$ & $\begin{array}{l}\text { Period of } \\
\text { Record }\end{array}$ & $\begin{array}{c}\text { Constituents } \\
\text { analyzed }\end{array}$ \\
\hline 42 & - & Croton 4 & LK-RES & 1984-present & BONP \\
\hline 43 & - & Croton 3 & LK-RES & 1984-present & BONP \\
\hline 44 & - & Croton 2 & LK-RES & 1984-present & BONP \\
\hline 45 & - & Croton 1 & LK-RES & 1984-present & BONP \\
\hline 46 & 412622073442600 & Edgewood Club Dist Syst-China Lake 1920d & LK-RES & $09-19-72$ & BOP \\
\hline 47 & 412451073411700 & Carmel Wd \#2 Dist Syst-Lake Glinda 0189d & LK-RES & 09-19-72 & BOP \\
\hline 48 & 412401073375500 & Brewster Heights Ds-middle Branch Res 1295d & LK-RES & $12-10-73$ & BOP \\
\hline 49 & 412152073345400 & Peach Lake at Peach Lake NY & LK-RES & $\begin{array}{c}07-07-76 \text { to } \\
11-17-76\end{array}$ & BONP \\
\hline 50 & 412232073472601 & $\begin{array}{l}\text { Carmel Water District No } 3 \text { WTP-Lake Secor } \\
0190 \mathrm{t}\end{array}$ & LK-RES & 01-16-75 & BOP \\
\hline 51 & 412225073471601 & Carmel Wd\#3 WTP-Lake Secor 0190t & LK-RES & $10-19-71$ & BOP \\
\hline 52 & 412329073444300 & $\begin{array}{l}\text { Lk Mahopac Gardens Dist Syst-Lake Mahopac } \\
\text { 0192d }\end{array}$ & LK-RES & 09-19-72 & BOP \\
\hline 53 & 412333073443200 & $\begin{array}{l}\text { Lk Mahopac Woods Dist Syst-Lake Mahopac } \\
\text { 0194d }\end{array}$ & LK-RES & 09-19-72 & BOP \\
\hline 54 & 412233073450200 & Mahopac Hills Dist Syst-Lake Mahopac 0198d & LK-RES & $09-19-72$ & BOP \\
\hline 55 & 412214073445100 & Lake Gardens Dist Syst-Lake Mahopac 0713d & LK-RES & $09-19-72$ & BOP \\
\hline 56 & 412217073443600 & Lake View Park Dist Syst-Lake Mahopac 0195d & LK-RES & 09-19-72 & BOP \\
\hline 57 & 412228073433800 & Lake Mahopac Ridge WTP-Lake Mahopac 0193t & LK-RES & 04-24-75 & BOP \\
\hline 58 & 01374910 & Lake Mahopac Ridge WTP-Lake Mahopac 0193r & LK-RES & 04-24-75 & BOP \\
\hline 59 & 412223073434200 & $\begin{array}{l}\text { Lk Mahopac Ridge Dist Syst-Lake Mahopac } \\
\text { 0193d }\end{array}$ & LK-RES & $09-19-72$ & BOP \\
\hline 60 & 412204073441000 & Mahopac Point Dist Syst-Lake Mahopac 0201d & LK-RES & $09-19-72$ & BOP \\
\hline 61 & 412024073434000 & Lake Lincolndale at Lincolndale NY & LK-RES & $\begin{array}{l}06-29-76 \text { to } \\
10-21-76\end{array}$ & BONP \\
\hline 62 & 411436073325000 & Lake Kitchawan at Lake Kitchawan & LK-RES & $\begin{array}{c}07-06-76 \text { to } \\
11-17-76\end{array}$ & BONP \\
\hline 63 & 411713073391700 & Lake Katonah at Lake Katonah NY & LK-RES & $\begin{array}{c}07-01-76 \text { to } \\
10-21-76\end{array}$ & BONP \\
\hline 64 & 412152073345401 & Peach Lake at Peach Lake NY & LK-RES & $07-07-76$ & BONP \\
\hline 65 & - & West Branch Release & SW & 1987-present & BONP \\
\hline 66 & - & Middle Branch Release & SW & 1987-present & BONP \\
\hline 67 & - & East Branch River & SW & 1987-present & BONP \\
\hline 68 & - & Bog Brook Release & SW & 1987-present & BONP \\
\hline 69 & - & Diverting Release & SW & 1987-present & BONP \\
\hline 70 & - & Kirk Lake Release & SW & 1987-present & BONP \\
\hline 71 & 一 & Croton Falls Release & SW & 1987-present & BONP \\
\hline 72 & - & Titicus River & SW & 1987-present & BONP \\
\hline 73 & - & Titicus Release & SW & 1987-present & BONP \\
\hline 74 & - & Muscoot River & sw & 1987-present & BONP \\
\hline 75 & - & Hallocks Mill Brook \#2 & sw & 1987-present & BONP \\
\hline 76 & - & Cross River & sw & 1987-present & BONP \\
\hline 77 & - & Cross River Release & sw & 1987-present & BONP \\
\hline 78 & - & Muscoot Release & SW & 1987-present & BONP \\
\hline
\end{tabular}


Table 3. (Continued) Summary of data collected at water-quality sites

\begin{tabular}{|c|c|c|c|c|c|}
\hline $\begin{array}{l}\text { No. on } \\
\text { fig. } 6\end{array}$ & $\begin{array}{c}\text { Site- } \\
\text { identification } \\
\text { number }\end{array}$ & Site name & $\begin{array}{l}\text { Type of } \\
\text { site }\end{array}$ & $\begin{array}{l}\text { Period of } \\
\text { Record }\end{array}$ & $\begin{array}{l}\text { Constituents } \\
\text { analyzed }\end{array}$ \\
\hline 79 & - & Beaver Dam Brook & SW & 1987-present & BONP \\
\hline 80 & - & Kisco River \#2 & SW & 1987-present & BONP \\
\hline 81 & - & Kisco River \#1 & SW & 1987-present & BONP \\
\hline 82 & - & Croton Release & SW & 1987-present & BONP \\
\hline 83 & - & Amawalk Release & SW & 1987-present & BONP \\
\hline 84 & - & Hallocks Mill Brook \#1 & SW & 1987-present & BONP \\
\hline 85 & - & East Branch Release & SW & 1987-present & BONP \\
\hline 86 & - & Boyd's Release & SW & 1987-present & BONP \\
\hline 87 & - & Middle Branch River & SW & 1987-present & BONP \\
\hline 88 & 01374540 & Holly Stream Near Brewster NY & SW & $07-14-70$ & QBO \\
\hline 89 & 01374545 & East Branch Croton River at Croton Falls NY & SW & 08-05-76 & QBONP \\
\hline 90 & 01374705 & West Branch Croton River at Croton Falls NY & SW & 08-05-76 & QBONP \\
\hline 91 & 01374930 & Muscoot River at Baldwin Place NY & SW & $\begin{array}{c}06-28-76 \text { to } \\
08-06-76\end{array}$ & QBONP \\
\hline 92 & 01374937 & Lake Shenorock Outlet at Shenorock NY & SW & 08-04-76 & QBONP \\
\hline 93 & 01374860 & Plum Brook at Lincolndale NY & SW & $\begin{array}{c}11-09-64 \text { to } \\
08-05-76\end{array}$ & QBONP \\
\hline 94 & 01374840 & Muscoot Reservoir Tributary at Somers NY & SW & 08-05-76 & QBONP \\
\hline 95 & 01374788 & Crook Brook at Salem Center NY & SW & 08-05-76 & QBONP \\
\hline 96 & 01374780 & Titicus River at Salem Center NY & SW & $\begin{array}{c}06-28-76 \text { to } \\
08-05-76\end{array}$ & QBONP \\
\hline 97 & 01374830 & Croton River Tributary at Goldens Bridge NY & SW & 08-04-76 & QBONP \\
\hline 98 & 01374880 & Waccabuc River at Boutonville NY & SW & $\begin{array}{c}07-07-76 \text { to } \\
08-04-76\end{array}$ & QBONP \\
\hline 99 & 01374976 & Angle Fly Brook at Whitehall Corners NY & SW & 08-04-76 & QBONP \\
\hline 100 & 01374992 & Hunter Brook Near Yorktown NY & SW & $\begin{array}{c}08-04-76 \text { to } \\
09-01-76\end{array}$ & QBONP \\
\hline 101 & 411707073500900 & Mill Pond Trib Off Hunter Brook Rd. at Yorktown & SW & $08-05-76$ & $\mathbf{n} / \mathbf{a}$ \\
\hline 102 & 01374993 & $\begin{array}{l}\text { Hunter Brook Below Mill Pond Near Yorktown } \\
\text { NY }\end{array}$ & SW & $\begin{array}{l}07-06-76 \text { to } \\
09-01-76\end{array}$ & QBONP \\
\hline 103 & 01374948 & Crom Pond Outlet at Yorktown Heights NY & Sw & 08-04-76 & QBONP \\
\hline 104 & 01374960 & Hallocks Mill Brook at Yorktown Heights NY & SW & $\begin{array}{l}07-06-76 \text { to } \\
07-21-76\end{array}$ & QBNP \\
\hline 105 & 01374963 & Hallocks Mill Brook at Amawalk NY & sw & $\begin{array}{c}07-19-76 \text { to } \\
08-04-76\end{array}$ & QBNP \\
\hline 106 & 01374942 & Muscoot River at Amawalk NY & SW & $\begin{array}{c}07-06-76 \text { to } \\
07-21-76\end{array}$ & QBNP \\
\hline 107 & 01374919 & Stone Hill River at Katonah NY & SW & $\begin{array}{l}06-28-76 \text { to } \\
08-05-76\end{array}$ & QBONP \\
\hline 108 & 01374917 & Broad Brook at Katonah NY & SW & $08-05-76$ & QBONP \\
\hline 109 & 01374908 & Stone Hill River at Bedford NY & SW & $\begin{array}{l}07-07-76 \text { to } \\
08-04-76\end{array}$ & QBONP \\
\hline 110 & 411218073434700 & Kisco River Trib at Green St. at Mount Kisco NY & Sw & $\begin{array}{l}08-04-76 \text { to } \\
11-16-76\end{array}$ & $\mathbf{n} / \mathbf{a}$ \\
\hline 111 & 01374983 & Kisco River at Lexington Ave. at Mount Kisco NY & SW & $\begin{array}{c}07-07-76 \text { to } \\
08-04-76\end{array}$ & QBONP \\
\hline
\end{tabular}


Table 3. (Continued) Summary of data collected at water-quality sites

\begin{tabular}{|c|c|c|c|c|c|}
\hline $\begin{array}{l}\text { No. on } \\
\text { fig. } 6\end{array}$ & $\begin{array}{c}\text { Site- } \\
\text { identification } \\
\text { number }\end{array}$ & Site name & $\begin{array}{l}\text { Type of } \\
\text { site }\end{array}$ & $\begin{array}{l}\text { Period of } \\
\text { Record }\end{array}$ & $\begin{array}{l}\text { Constituents } \\
\text { analyzed }\end{array}$ \\
\hline 112 & 01374987 & Kisco River Below Mount Kisco NY & SW & $\begin{array}{c}07-07-76 \text { to } \\
08-04-76\end{array}$ & QBONP \\
\hline 113 & 01374988 & Gedney Brook Near Mount Kisco NY & SW & $08-04-76$ & QBONP \\
\hline 114 & 01375000 & $\begin{array}{l}\text { Croton R @ New Croton Dam near Croton-on- } \\
\text { hudson NY }\end{array}$ & SW & $\begin{array}{c}10-06-70 \text { to } \\
06-21-72\end{array}$ & QBON \\
\hline 115 & 01374890 & Cross River Near Cross River NY & SW & $\begin{array}{c}06-28-76 \text { to } \\
08-05-76\end{array}$ & QBONP \\
\hline 116 & 01374970 & Muscoot River Near Amawalk NY & SW & $\begin{array}{c}07-06-76 \text { to } \\
07-21-76\end{array}$ & QBNP \\
\hline 117 & 01374494 & Haviland Hollow Brook Near Putnam Lake NY & SW & $07-14-70$ & QBO \\
\hline 118 & 01374965 & Hallocks Mill Brook Below Amawalk NY & SW & $\begin{array}{c}07-06-76 \text { to } \\
07-20-76\end{array}$ & Q \\
\hline 119 & 412757073425800 & Putnam 30 & GW & $01-15-75$ & BOP \\
\hline 120 & 412757073425801 & Putnam 900 & GW & 01-14-75 & BOP \\
\hline 121 & 412644073410400 & Putnam 28 & GW & $01-21-75$ & BOP \\
\hline 122 & 412405073360400 & Putnam 26 & GW & $10-19-72$ & BOP \\
\hline 123 & 412504073325800 & Putnam 27 & GW & $09-19-72$ & BOP \\
\hline 124 & 412212073350800 & Putnam 23 & GW & $01-16-75$ & BOP \\
\hline 125 & 412125073331801 & Westchester 3864 & GW & $\begin{array}{c}10-17-86 \text { to } \\
05-06-87\end{array}$ & BON \\
\hline 126 & 412025073344401 & Westchester 3865 & GW & $\begin{array}{c}10-17-86 \text { to } \\
05-06-87\end{array}$ & BON \\
\hline 127 & 412012073334701 & Westchester 3862 & GW & $\begin{array}{c}10-17-86 \text { to } \\
05-06-87\end{array}$ & BON \\
\hline 128 & 411935073340801 & Westchester 3861 & GW & $\begin{array}{c}10-17-86 \text { to } \\
05-06-87\end{array}$ & BON \\
\hline 129 & 411900073370101 & Westchester 3863 & GW & $\begin{array}{c}10-17-86 \text { to } \\
05-06-87\end{array}$ & BON \\
\hline 130 & 411651073342101 & Westchester 3856 & GW & $\begin{array}{c}10-17-86 \text { to } \\
05-05-87\end{array}$ & BON \\
\hline 131 & 411627073325701 & Westchester 3857 & GW & $\begin{array}{c}10-17-86 \text { to } \\
05-05-87\end{array}$ & BON \\
\hline 132 & 411542073323601 & Westchester 3858 & GW & $10-17-86$ & BON \\
\hline 133 & 411544073370301 & Westchester 3855 & GW & $10-17-86$ & BON \\
\hline 134 & 411532073364801 & Westchester 3854 & GW & $\begin{array}{l}10-17-86 \text { to } \\
05-05-87\end{array}$ & BON \\
\hline 135 & 412037073400001 & Westchester 3869 & GW & $\begin{array}{c}10-15-86 \text { to } \\
05-04-87\end{array}$ & BON \\
\hline 136 & 411915073400701 & Westchester 3870 & GW & $\begin{array}{c}10-15-86 \text { to } \\
05-05-87\end{array}$ & BOP \\
\hline 137 & 411839073393301 & Westchester 3860 & GW & $\begin{array}{l}10-15-86 \text { to } \\
05-04-87\end{array}$ & BON \\
\hline 138 & 411736073400301 & Westchester 3853 & GW & $\begin{array}{l}10-15-86 \text { to } \\
05-04-87\end{array}$ & BON \\
\hline 139 & 411618073395401 & Westchester 5010 & GW & $\begin{array}{c}10-15-86 \text { to } \\
05-04-87\end{array}$ & BON \\
\hline
\end{tabular}


Table 3. (Continued) Summary of data collected at water-quality sites

\begin{tabular}{|c|c|c|c|c|c|}
\hline $\begin{array}{c}\text { No. on } \\
\text { fig. } 6\end{array}$ & $\begin{array}{c}\text { Site- } \\
\text { identification } \\
\text { number }\end{array}$ & Site name & $\begin{array}{c}\text { Type of } \\
\text { site }\end{array}$ & $\begin{array}{c}\text { Period of } \\
\text { Record }\end{array}$ & $\begin{array}{c}\text { Constituents } \\
\text { analyzed }\end{array}$ \\
\hline 140 & 411942073411201 & Westchester 3871 & GW & $\begin{array}{c}10-15-86 \text { to } \\
05-05-87\end{array}$ & BON \\
\hline 141 & 411947073415301 & Westchester 3868 & GW & $\begin{array}{c}10-15-86 \text { to } \\
05-06-87\end{array}$ & BON \\
\hline 142 & 412127073462700 & Putnam 8 & GW & $12-10-73$ & BOP \\
\hline 143 & 412045073455300 & Putnam 6 & GW & $01-16-75$ & BOP \\
\hline 144 & 411917073431200 & Westchester 40 & GW & $03-05-74$ & BOP \\
\hline 145 & 411832073454201 & Westchester 3872 & GW & $\mathrm{n} / \mathrm{a}$ & $\mathrm{n} / \mathrm{a}$ \\
\hline 146 & 411629073423901 & Westchester 3873 & GW & $\begin{array}{c}10-15-86 \text { to } \\
05-06-87\end{array}$ & BON \\
\hline 147 & 411433073434901 & Westchester 5012 & GW & $\begin{array}{c}10-16-86 \text { to } \\
05-05-87\end{array}$ & BON \\
\hline 148 & 411429073414800 & Westchester 39 & GW & $\begin{array}{c}12-08-71 \text { to } \\
03-05-74\end{array}$ & BOP \\
\hline 149 & 411405073370001 & Westchester 5017 & GW & $\begin{array}{c}10-16-86 \text { to } \\
05-05-87\end{array}$ & BON \\
\hline 150 & 411222073363201 & Westchester 5018 & GW & $\begin{array}{c}10-16-86 \text { to } \\
05-06-87\end{array}$ & BON \\
\hline 151 & 411308073410001 & Westchester 5015 & GW & $\begin{array}{c}10-16-86 \text { to } \\
05-05-87\end{array}$ & BON \\
\hline 152 & 411349073393701 & Westchester 5016 & GW & $\begin{array}{c}10-16-86 \text { to } \\
05-05-87\end{array}$ & BON \\
\hline 153 & 411116073422301 & Westchester 4424 & GW & $\begin{array}{c}10-16-86 \text { to } \\
05-06-87\end{array}$ & BON \\
\hline 154 & 410958073433201 & Westchester 3866 & GW & $\begin{array}{c}10-16-86 \text { to } \\
05-05-87\end{array}$ & BON \\
\hline 155 & 411144073440900 & Westchester 36 & GW & $12-08-71$ & BOP \\
\hline 156 & 411317073435601 & Westchester 5011 & GW & $\begin{array}{c}10-16-86 \text { to } \\
05-05-87\end{array}$ & BON \\
\hline 157 & 411411073461901 & Westchester 2095 & GW & $08-22-88$ & BO \\
\hline 158 & 411522073484401 & Westchester 2113 & GW & $08-24-88$ & BO \\
\hline 159 & 411458073492901 & Westchester 2094 & GW & $08-22-88$ & BO \\
\hline 160 & 411546073502501 & Westchester 2107 & GW & $08-24-88$ & BO \\
\hline 161 & 411539073503201 & Westchester 2106 & GW & $08-24-88$ & BO \\
\hline 162 & 411600073512201 & Westchester 2108 & GW & $08-24-88$ & BO \\
\hline 163 & 411310073494901 & Westchester 2097 & GW & $08-22-88$ & BO \\
\hline 164 & 411240073493801 & Westchester 2096 & GW & $\mathrm{n} / \mathrm{a}$ & $\mathrm{n} / \mathrm{a}$ \\
\hline 165 & 411947073442801 & Westchester 1460 & GW & $12-08-71$ & BOP \\
\hline 166 & 412156073441300 & Putnam 16 & GW & $02-05-75$ & BOP \\
\hline 167 & 412308073454600 & Putnam 24 & GW & $01-16-75$ & BOP \\
\hline 168 & 411947073442800 & Westchester 41 & GW & $12-08-71$ & BOP \\
\hline 169 & 411643073500101 & Westchester 1132 & GW & $08-25-88$ & BO \\
\hline 170 & 411946073443001 & Westchester 3867 & GW & $\begin{array}{c}10-15-86 \text { to } \\
05-05-87\end{array}$ & BON \\
\hline 171 & 412127073462701 & Putnam 9 & GW & $12-10-73$ & BOP \\
\hline 172 & NY0208027 & Amawalk-Shenorock Water Dist. & SW & $\mathrm{n} / \mathrm{a}$ & $\mathrm{n} / \mathrm{a}$ \\
\hline
\end{tabular}


Table 3. (Continued) Summary of data collected at water-quality sites

\begin{tabular}{|c|c|c|c|c|c|}
\hline $\begin{array}{l}\text { No. on } \\
\text { fig. } 6\end{array}$ & $\begin{array}{c}\text { Site- } \\
\text { identification } \\
\text { number }\end{array}$ & Site name & $\begin{array}{c}\text { Type of } \\
\text { site }\end{array}$ & $\begin{array}{l}\text { Period of } \\
\text { Record }\end{array}$ & $\begin{array}{l}\text { Constituents } \\
\text { analyzed }\end{array}$ \\
\hline 173 & NY0026743 & Yorktown (T) & SW & $\mathrm{n} / \mathrm{a}$ & $\mathrm{n} / \mathrm{a}$ \\
\hline 174 & NY0026727 & Yorktown (T) & SW & $\mathrm{n} / \mathrm{a}$ & $\mathrm{n} / \mathrm{a}$ \\
\hline 175 & NY0149195 & Croton-Harmon School District & SW & $\mathrm{n} / \mathrm{a}$ & $\mathrm{n} / \mathrm{a}$ \\
\hline 176 & NY0149195 & Croton-Harmon School District & SW & $\mathrm{n} / \mathrm{a}$ & $\mathrm{n} / \mathrm{a}$ \\
\hline 177 & NY0207969 & Kent Elementary School & SW & $\mathrm{n} / \mathrm{a}$ & $\mathrm{n} / \mathrm{a}$ \\
\hline 178 & NY0031356 & Carmel (T) & SW & $\mathrm{n} / \mathrm{a}$ & $\mathbf{n} / \mathbf{a}$ \\
\hline 179 & NY0026581 & NYC Dept. Env Protection & SW & $\mathrm{n} / \mathrm{a}$ & n/a \\
\hline 180 & NY0026590 & NYC Dept. Env. Protection-Mahopac & SW & $\mathrm{n} / \mathrm{a}$ & n/a \\
\hline 181 & NY0208027 & Amawalk-Shenorock Water Dist. & SW & $\mathrm{n} / \mathrm{a}$ & $\mathrm{n} / \mathrm{a}$ \\
\hline 182 & NY0233625 & Spain Oil & SW & $\mathrm{n} / \mathrm{a}$ & $\mathrm{n} / \mathrm{a}$ \\
\hline 183 & - & Croton-raw & $\begin{array}{c}\text { KEY } \\
\text { POINT }\end{array}$ & 1987-present & BONP \\
\hline
\end{tabular}

use data for the study were obtained from a variety of sources. Putnam County data were obtained from the NYCDEP as a polygon data set at the scale of $1: 24,000$, interpreted from 1987 aerial photographs and updated in 1991 with field checking; this data set represented about 50 land-use categories. Westchester County data were obtained from the Westchester County Department of Planning as a polygon data set at a scale of $1: 24,000$ containing land-use information from the early 1980's; this data set contained 13 landuse categories. Data for Dutchess County and the parts of the study area that are in Fairfield County, Conn., were obtained from the USGS Geographic Information Retrieval and Analysis System (GIRAS) data base, which represents data from the mid-1970's and includes more than 30 land-use categories. Land-use categories that are found in the above data sets include low-, medium-, and high-density residential development; transportation corridors; commercial; industrial; cropland; orchards; deciduous forest land; quarries; light manufacturing; inactive urban; and shopping centers. For the purpose of this study, all land-use categories were combined into four general groups - undeveloped land, residential land, agricultural land, and commercial land (including industrial areas and transportation corridors, fig. 7).

The dominant land-use category in the study area is undeveloped land which represents $\mathbf{5 6 . 7}$ percent of the study area. The next largest land-use category is residential land ( 25.3 percent of the study area); although it is present throughout the study area, it is concentrated primarily around transportation corridors, reservoirs, and lakes. The next largest category is agricultural land (7.4 percent of the study area); this category is primarily in the northern and eastern parts of the study area. Commercial land use (4.1 percent of the study area) is concentrated mainly west of the Croton River, between the Muscoot Reservoir and the Croton Falls Reservoir, but also is found along transportation corridors. Lakes and reservoirs (5.7 percent of the study area) and undetermined land use ( 0.8 percent of the study area) occupy the rest of the study area.

Potential point-source-contamination sites include active and inactive hazardous-waste sites within the study area; data on these sites are listed in table 4. The data were obtained from the NYSDEC, Region 3 (NYSDEC, 1992). The study area contains 24 potential point-source-contamination sites; two are on the USEPA National Priorities List-one in Putnam County and one in Westchester County.

\section{POTENTIAL MONITORING SITES}

Sites for a water-resources monitoring network in the Croton Reservoir system were selected subjectively with the aid of the GIS coverages described 


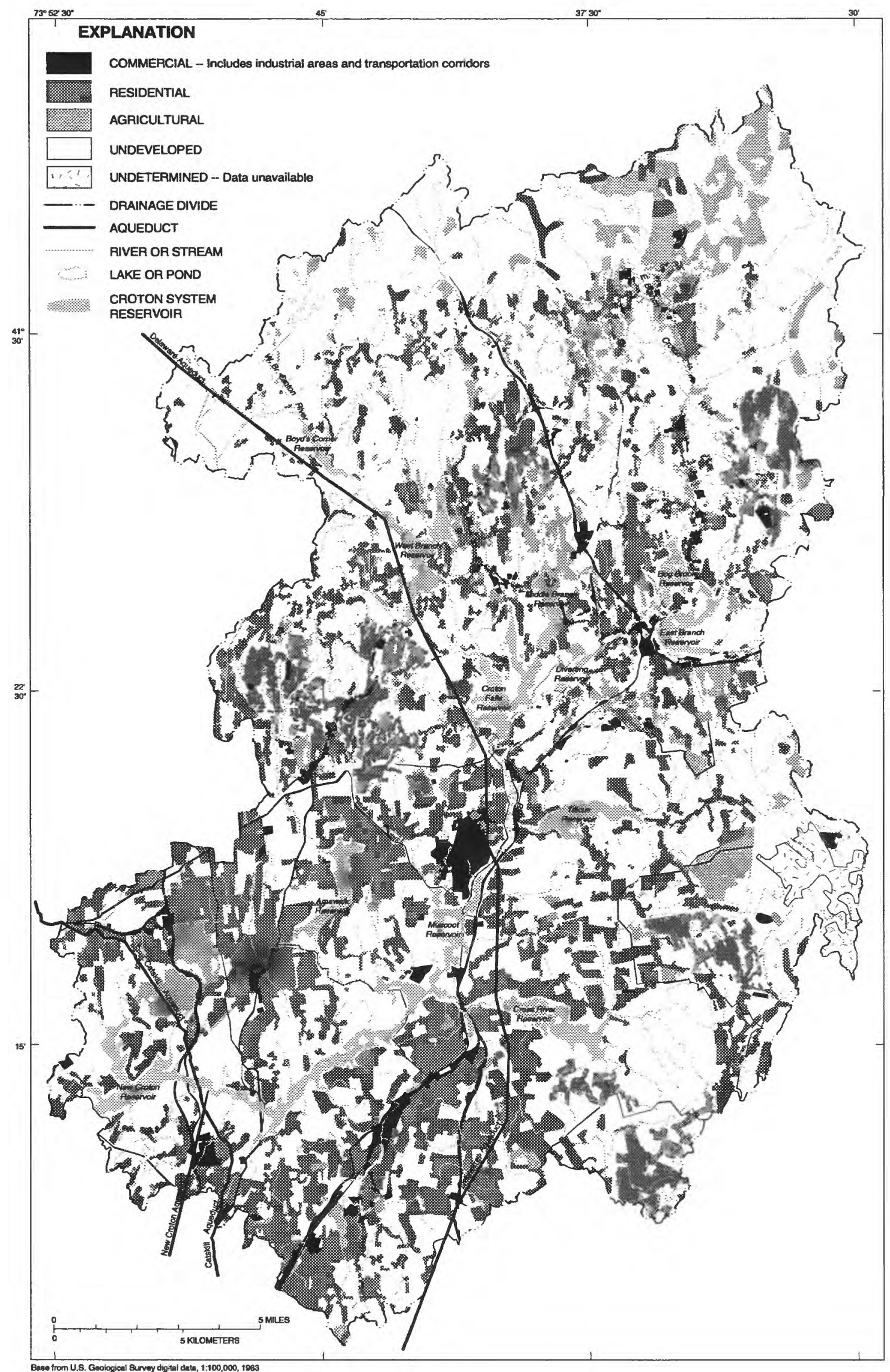

Figure 7. Land use within the Croton Reservoir system. 
Table 4. Sites that are potential point-sources of contamination in southern Dutchess, Putnam, and northern Westchester Counties, N.Y.

[-, unknown; GW, ground water; SW, surface water; DW, drinking water. Data from New York State Department of Environmental Conservation.]

\begin{tabular}{|c|c|c|c|c|}
\hline Owner & County & Town & Type of site & $\begin{array}{l}\text { Resource } \\
\text { affected }\end{array}$ \\
\hline Pawling Rubber Co. & Dutchess & Pawling & Landfill & GW/DW \\
\hline Texaco Research Center & Dutchess & East Fishkill & Lagoon/Landfill & GW \\
\hline Royal Carting Service & Dutchess & East Fishkill & Open dump & - \\
\hline Old Beekman Site & Dutchess & Beekman & Open dump & - \\
\hline Pawling Village landfill & Dutchess & Pawling & Landfill & - \\
\hline Hopewell Precision Inc. & Dutchess & East Fishkill & Open dump & - \\
\hline IBM-East Fishkill & Dutchess & East Fishkill & Open dump & GW \\
\hline East Fishkill Landfill & Dutchess & East Fishkill & Landfill & GW \\
\hline Metro North (Conrail-Harlem Div.) & Putnam & Southeast & Open dump & - \\
\hline Champion Building Products & Putnam & Patterson & Open dump & GW/DW \\
\hline Cross Co. Sanitary-Kessman L.F. & Putnam & Patterson & Landfill & GW/Air \\
\hline Brewster Village Well Field 1 & Putnam & Southeast & Open dump & GW \\
\hline Mahopac Business District Wells & Putnam & Carmel & Open dump & GW/DW \\
\hline Croton Point Sanitary Landfill & Westchester & Croton-on-Hudson & Landfill & GW/SW \\
\hline Magna Metals & Westchester & Cortlandt & - & SW \\
\hline Armonk Private Wells & Westchester & North Castle & Open dump & GW/SW/DW \\
\hline Bedford Village Wells-Shopping Arcade & Westchester & Bedford & Open dump & - \\
\hline Katonah Well (Bed Water Stor. \& Dist.) ${ }^{1}$ & Westchester & Bedford & Open dump & GW/DW \\
\hline Bedford Village Wells-Hunting Ridge Mall & Westchester & Bedford & Open dump & GW/DW \\
\hline Harmon Railroad Yard-Waste Water Area & Westchester & Croton & Lagoon & - \\
\hline Westchester Colprovia Corporation & Westchester & Bedford & Structure & GW \\
\hline Harmon Railroad Yard-Metro North RR & Westchester & Croton & - & GW \\
\hline Baldwin Place Shopping Center & Westchester & Somers & Structure & GW/DW \\
\hline Marx Residence & Westchester & New Castle & Structure & GW/DW \\
\hline
\end{tabular}

${ }^{1}$ on the USEPA National Priorities List

earlier. Three types of data-collection sites were identified that, together, would provide adequate waterresources monitoring for the basin: reservoir-outflowmonitoring sites (to document rate and chemical quality of reservoir outflow), reservoir-inflowmonitoring sites (to document rate and chemical quality of reservoir inflow), and ground-water-monitoring areas (to document ground-water levels and chemical quality of ground water).

Reservoir-inflow and -outflow rates can be used to calculate the change in reservoir storage for a given period, and ground-water levels at wells provide information on seasonal long-term trends in the major aquifers throughout the study area. The water-level data also can be used to (1) calculate the amount of water available from aquifers that provide base flow to streams, and (2) monitor long-term trends in water use and aquifer recharge.

The following paragraphs describe the subjective selection of monitoring sites for reservoir-outflow, reservoir-inflow, and ground-water-monitoring areas. The GIS coverages that were used in the selection of each of these three data categories are given in table 5 .

\section{Reservoir-Outflow Sites}

Reservoir-outflow sites would be established either at a dam that forms the reservoir or at the closest site downstream that is suitable for a streamflow gage. Outflow data, when combined with inflow data, can be used to calculate chemical and nutrient loading and 
calculate the change in storage of the reservoir. Establishment of outflow-measurement sites at several dams throughout the Croton Reservoir system would enable calculation of change in storage and of chemical and nutrient loading within the watershed. GIS coverages that were used in the selection of locations for reservoiroutflow-monitoring sites are given in table 5. The coverages "surface-water gages" and "water-quality sites" were used to identify which reservoirs had long-term data that could (1) provide information on historical trends, and (2) be incorporated with new data-collection efforts. The "diversion," "dams," "public-water-supply withdrawals," and "water-quality point sources" coverages were used to identify areas that have been affected by man. Data-collection sites would be installed downstream from reservoirs to provide data on the quantity and quality of outflow. The "hydrography" and "basins" coverages provided the information needed to delineate the boundaries of the drainage areas represented by each reservoir-outflow site. Eight reservoir-outflow sites (fig. 8) were selected to provide data on the flow and quality of water discharging from the reservoirs; all are at reservoirs and dams maintained by NYCDEP - West Branch, Bog Brook and East Branch (combined), Diverting, Croton Falls, Titicus, Cross River, Amawalk, and Muscoot. Data on their drainage areas are given in table 6.

\section{Reservoir-Inflow Sites}

The second group of data-collection sites that was subjectively selected included sites on unregulated streams or rivers that discharge to reservoirs in the drainage basins of the Croton Reservoir system. This group provides information on the inflow to reservoirs and, when combined with reservoir-outflow data, can be used to calculate change in storage. Each drainage basin in which a reservoir-inflow site was under consideration was assigned two dominant landuse categories to allow the streamflow and waterquality data from these sites to be projected to similar ungaged sites in areas that represent other basins.

Selection of basins for reservoir-inflow-monitoring sites was based primarily on subbasin boundaries obtained from the GIS "basins" coverage and on the land use within the headwater basin, as indicated by the "land use" coverage. Originally, the basins considered for reservoir-inflow-monitoring were chosen to represent only areas that were either rural or urban, but basins with other secondary land uses were later allowed because most of the basins had other dominant land uses. Additional considerations in the subjective selection of basins for reservoir-inflow monitoring were: population distribution, potential hazardouswaste sites, long-term hydrologic data collected by NYCDEP, transferability of observed trends in flow and water-quality characteristics to other parts of the study area, and the spatial distribution of the selected basins.

Twelve GIS coverages were used in the selection of reservoir-inflow basins (table 5). Initially, the "basins" coverage was overlain by the "land use" coverage to indicate subbasins with only one or two major land uses; the remaining coverages were then used to further differentiate the subbasins.

Nine reservoir-inflow sites representing a combination of four major land uses were finally selected (fig. 9); the primary and secondary land uses and the drainage area of each basin are given in table 7. All of the four primary land-use categories in the Croton Reservoir system are represented in these nine basins. Information gathered in a gaged basin within this group is applicable to ungaged basins within the Croton Reservoir system that have similar land-use characteristics. Some of the basins were selected

Table 5. Monitoring categories to which each GIS coverage was applied in site-selection process in the Croton Reservoir system

\begin{tabular}{lccc}
\hline & \multicolumn{3}{c}{ Type of monitoring site } \\
\cline { 2 - 4 } \multicolumn{1}{c}{ GIS data set } & $\begin{array}{c}\text { Reservoir } \\
\text { outflow }\end{array}$ & $\begin{array}{c}\text { Reservoir } \\
\text { inflow }\end{array}$ & $\begin{array}{c}\text { Ground } \\
\text { water }\end{array}$ \\
\hline Basins & yes & yes & yes \\
Population & no & yes & yes \\
Land use & no & yes & yes \\
Roads & no & yes & yes \\
Hydrography & yes & yes & yes \\
Surface-water gages & yes & yes & no \\
Inflows and diversions & yes & yes & no \\
Bedrock geology & no & no & yes \\
Surficial geology & no & no & yes \\
Public-water-supply & yes & yes & yes \\
withdrawals & & & \\
Ground-water wells & no & no & yes \\
Water-quality sites & yes & yes & yes \\
Water-quality point & yes & yes & yes \\
source & & & yes \\
Dams & yes & no & yes \\
Land surface & & & \\
\hline & & yes & \\
\hline & & &
\end{tabular}




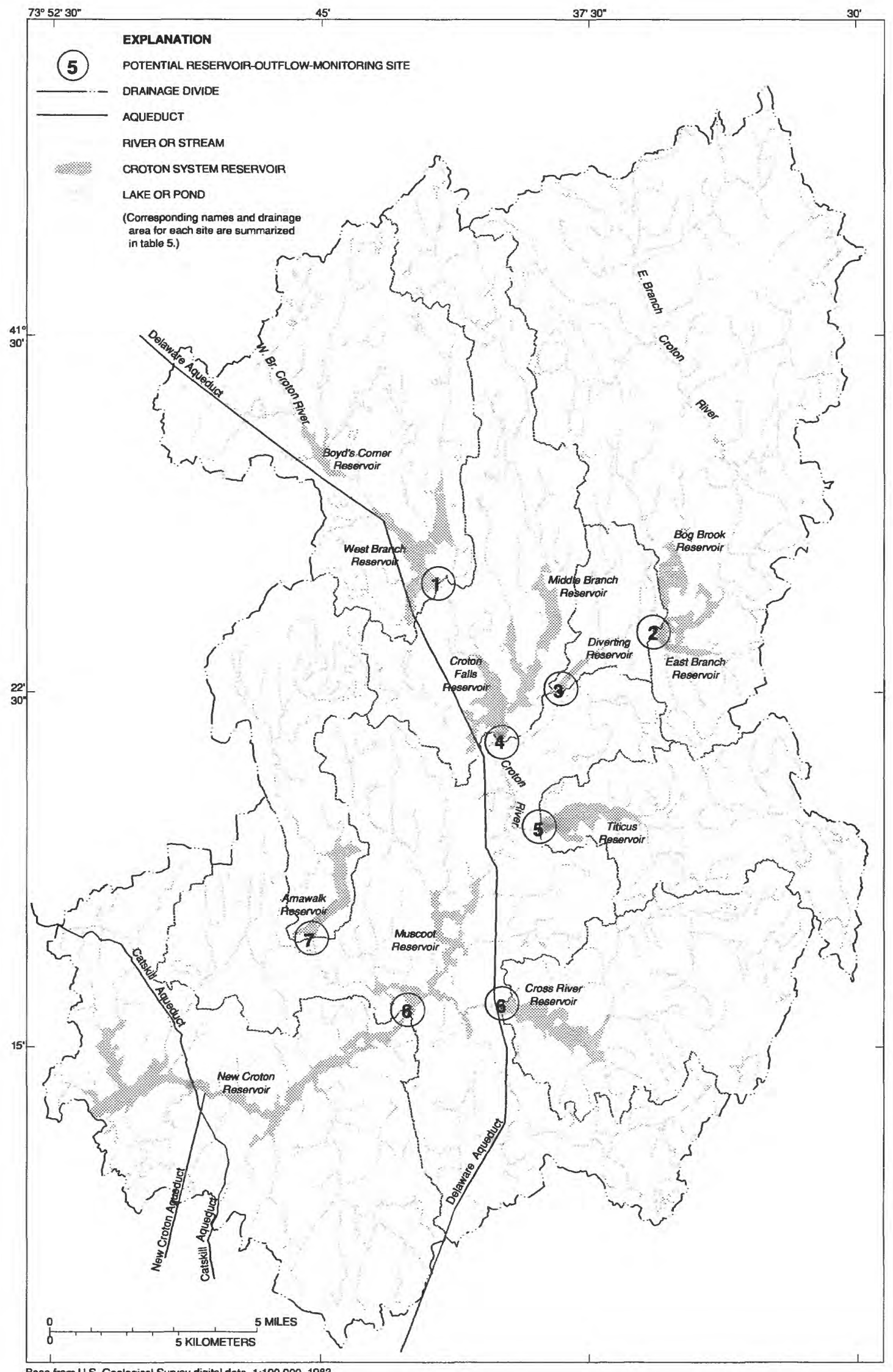

Figure 8. Potential monitoring sites at reservoir outflows in the Croton Reservoir system. 


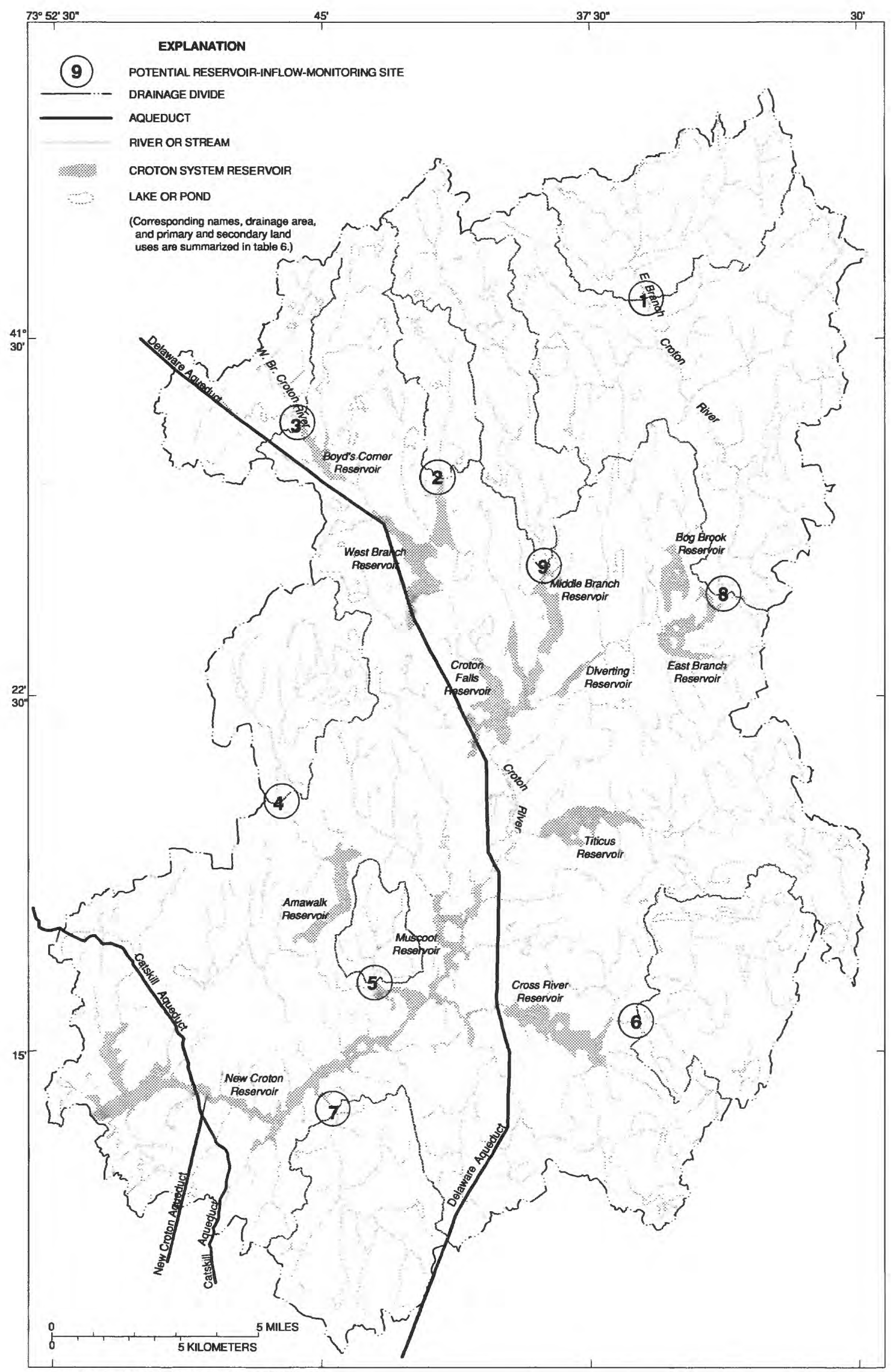

Base from U.S. Geological Survey digital data, 1:100,000, 1983

Figure 9. Potential monitoring sites in the headwaters of the Croton Reservoir system. 
because certain secondary criteria (such as size or population) would allow comparison of trends in waterresources characteristics; for example, Site 8 was selected because its large drainage area allows study of a wide range in flow trends, and site 9 was selected because it has a large population and, thus, could provide information on the effects of urban development.

Table 6.-Potential reservoir-outflow monitoring sites in the Croton Reservoir system

[Locations are shown in fig. 8. All drainage areas are in square miles.]

\begin{tabular}{clcc}
\hline $\begin{array}{c}\text { Site } \\
\text { number }\end{array}$ & Reservoir name & $\begin{array}{c}\text { Drainage area } \\
\text { Drainage } \\
\text { area at site }\end{array}$ & $\begin{array}{c}\text { notuded } \\
\text { with another } \\
\text { upstream site }\end{array}$ \\
\hline 1 & West Branch & 42.7 & 42.7 \\
2 & Bog Brook / & 79.4 & 79.4 \\
& East Branch & & \\
3 & Diverting & 86.4 & 7.00 \\
4 & Croton Falls & 169 & 39.9 \\
5 & Titicus & 23.4 & 21.9 \\
6 & Cross River & 29.9 & 31.4 \\
7 & Amawalk & 19.7 & 19.7 \\
8 & Muscoot & 319 & 72.8 \\
\hline
\end{tabular}

\section{Ground-Water-Monitoring Areas}

The third group of data-collection sites that was identified, those in ground-water-monitoring areas, included sites where an observation well could be installed for sample collection and water-level measurement. As with the reservoir-inflow sites, all possible ground-water-monitoring areas were first identified, and the smallest number that would adequately describe the ground-water quality and ground-water levels within the study area was subjectively chosen.

Twelve GIS coverages were used to identify the ground-water-monitoring areas (table 5); the boundaries of the previously identified reservoir-inflow basins also were used. The "bedrock geology" and "surficial geology" coverages were used to identify the formations and aquifers of greatest importance in the study area, and the monitoring areas were selected to represent each of the primary geologic formations and aquifers. A monitoring area also would be selected in each of the eight selected reservoir-inflow-monitoring basins so that each reservoir's basin would have both surface-water and ground-water data. Final selection resulted in 16 ground-water-monitoring areas in the Croton Reservoir system (fig. 10). Each area represents a different geologic unit and(or) represents an entire reservoir-inflow basin. The locations represented and primary reasons for selection for each ground-water basins are listed in table 8. Eight of the areas contain reservoir-inflow basins; five others represent major bedrock formations; and the remaining three represent surficial aquifers.

Each of the 16 ground-water monitoring areas will have one well - an index well from which groundwater-level data and water samples will be collected on a routine basis. The process for selection of an index well in each ground-water-monitoring area will be to first collect water samples from five wells in each area, then identify which of these wells best reflects the chemistry characteristics of ground water in that area and use that well as the index well for that area.

\section{Water-Quality-Monitoring Sites}

The objective of the water-resources-monitoring network is to collect water-quality data that can be used to indicate the effects of land use on the quality of surface water and ground water in the basin and to identify temporal or spatial trends. Routine collection of water samples from all data-collection sites would provide the data. Water quality was given high priority during the site-selection process.

All data-collection sites described previously also serve as sampling sites for water-quality analysis. Samples from the surface-water sites (reservoir-outflow and -inflow sites, figs. 8 and 9, respectively) would be analyzed for major cations and anions, trace metals, pesticides, and nutrients, and samples from wells would be analyzed for major cations and anions, trace metals, volatile organic compounds, pesticides, nutrients, and radon.

\section{SUMMARY}

Urbanization in the Croton River watershed in southeastern New York has led to the deterioration of water quality within the basin. A GIS data base was used in the site-selection process for a waterresources-monitoring network in the Croton Reservoir system. The monitoring network could enable water managers to detect future changes in the quality of surface water and ground water. 
Table 7. Sites selected for reservoir-inflow monitoring in the Croton Reservoir system

[Locations are shown in fig. 9. Drainage areas are in square miles.]

\begin{tabular}{clccc}
\hline \multirow{2}{*}{$\begin{array}{c}\text { Site } \\
\text { number }\end{array}$} & \multirow{2}{*}{ Headwater basin name } & $\begin{array}{c}\text { Drainage } \\
\text { area }\end{array}$ & \multicolumn{2}{c}{ Land use } \\
\cline { 4 - 5 } & & 17.3 & Primary & Secondary \\
\hline 1 & Upper East Branch Croton River & & Undeveloped & Undevelo \\
2 & Horse Pound Brook & 5.75 & Undeveloped & Residential \\
3 & Upper West Branch Croton River & 11.0 & Undeveloped & none \\
4 & Upper Muscoot River & 13.5 & Residential & Undeveloped \\
5 & Angle Fly Brook & 4.05 & Undeveloped & Residential \\
6 & Upper Cross River & 17.1 & Undeveloped & Residential \\
7 & Kisco River & 17.5 & Residential & Commercial \\
8 & East Branch Croton River ${ }^{1}$ & 63.2 & Undeveloped & Agriculture \\
9 & Middle Branch Croton River & 18.7 & Undeveloped & Residential \\
\hline
\end{tabular}

${ }^{1}$ This basin includes site 1.

Table 8. Potential ground-water-monitoring areas in the Croton Reservoir system

[Area-identification numbers are shown in fig. 10.]

\begin{tabular}{clcl}
\hline $\begin{array}{c}\text { Area- } \\
\text { identification } \\
\text { number }\end{array}$ & \multicolumn{1}{c}{ Location name } & $\begin{array}{c}\text { Size of area } \\
\text { represented }\end{array}$ & \\
(square miles) & \multicolumn{1}{c}{ Reason for selection } \\
\hline 1 & Upper East Branch Croton River & 17.3 & reservoir-inflow basin \\
2 & Horse Pound Brook & 5.75 & reservoir-inflow basin \\
3 & Upper West Branch Croton River & 11.0 & reservoir-inflow basin \\
4 & Upper Muscoot River & 13.5 & reservoir-inflow basin \\
5 & Angle Fly Brook & 4.05 & reservoir-inflow basin \\
6 & Upper Cross River & 17.1 & reservoir-inflow basin \\
7 & Kisco River & 17.5 & reservoir-inflow basin \\
8 & Middle Branch Croton River & 18.7 & reservoir-inflow basin \\
9 & Great Swamp, East Branch Croton River & 4.42 & major surficial aquifer \\
10 & Upper Stoney Brook & 1.48 & major surficial aquifer \\
11 & Central Somers & 1.02 & major surficial aquifer \\
12 & Southern Croton River basin & 11.7 & (Inwood marble, OEi) bedrock aquifer \\
13 & Southern Croton River basin & 73.7 & (Manhattan schist, Om) bedrock aquifer \\
14 & Southern Croton River basin & 27.3 & (Fordham gneiss, f) bedrock aquifer \\
15 & Eastern central Croton River basin & 28.0 & (Amphibolite, am) bedrock aquifer \\
16 & Northwestern Croton River basin & 59.9 & (Biotite-quartz-plagioclase gneiss, bqpc) \\
\hline & & & bedrock aquifer \\
\hline
\end{tabular}

\footnotetext{
${ }^{1}$ Bedrock areas overlap part of some reservoir-inflow basins.
} 


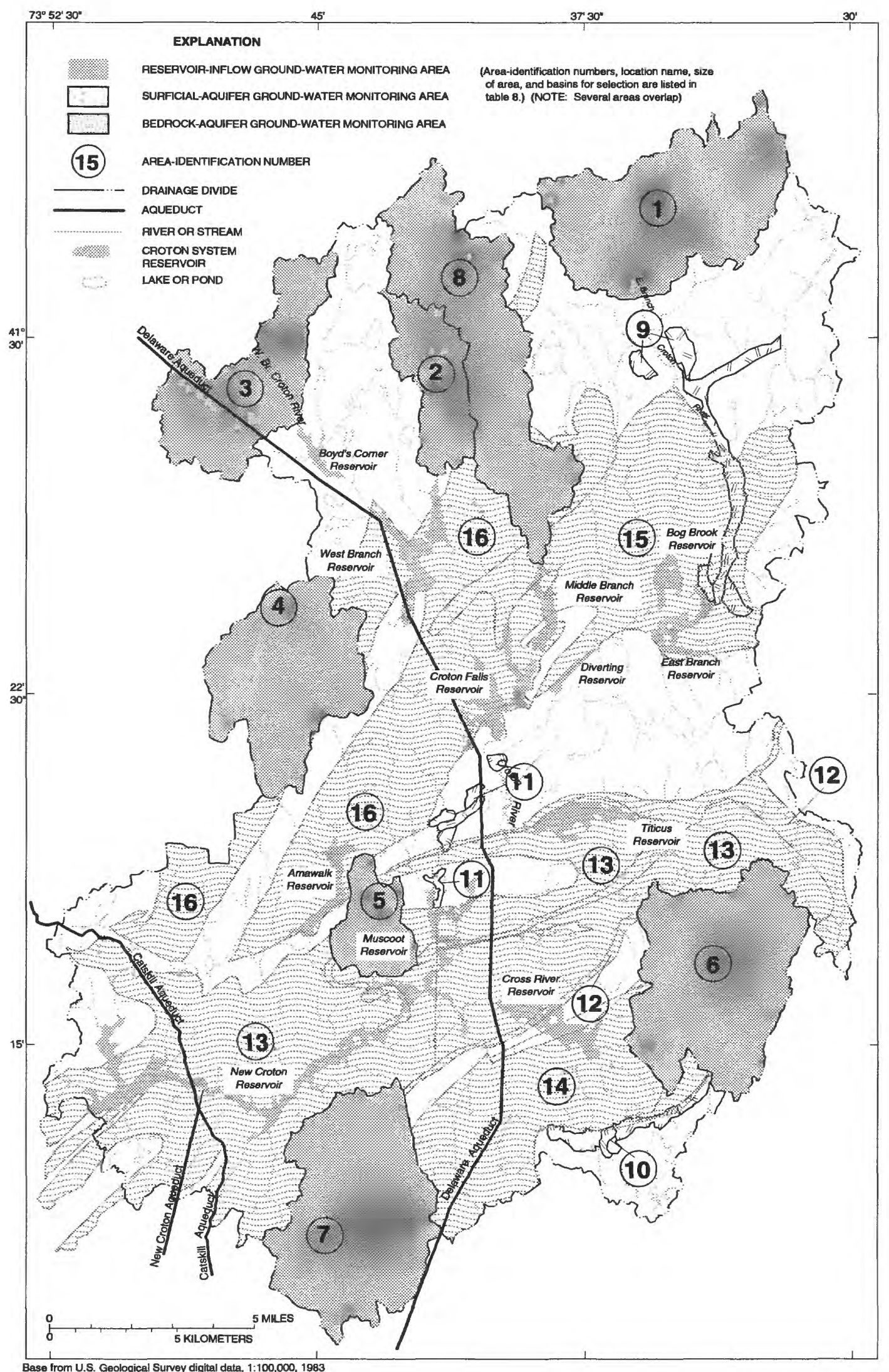

Figure 10. Potential ground-water monitoring areas in the Croton Reservoir system. 
The site-selection process consisted of two steps. The first was to develop a GIS data base of longterm and current water-resources data; the second was to apply the GIS data base to subjectively identify potential locations for measurement of surface-water discharge and ground-water levels and for sampling of surface water and ground water. The GIS data base consisted of 15 coverages "basins," "population," "land use," "roads," "hydrography," "surface-water gages," "inflows and diversions," "bedrock geology," "surficial geology," "public-water supply withdrawals," "ground water," "water-quality sites," "water-quality point sources," "dams," and "land surface." The GIS provided an efficient method of storing data for use in the identification of suitable locations for monitoring sites.

Three types of potential monitoring sites were subjectively identified: reservoir-outflow sites, to provide data on the rate of water flowing out of each reservoir; reservoir-inflow sites, to provide unregulated-streamflow data that could be transferred to ungaged sites; and ground-water monitoring areas, in which an index well would be established to monitor ground-water levels. Water samples would be collected at all surface-water and ground-water sites for chemical analyses, and the results would be used in conjunction with streamflow data and ground-water levels to evaluate and monitor all water resources of the Croton Reservoir system. Eight reservoir-outflow sites, nine reservoir-inflow sites, and 16 ground-water monitoring areas were subjectively selected.

\section{REFERENCES CITED}

Cadwell, D.H., Connally, G.G., Dineen, R.J., Fleisher, P.J., Fuller, M.L., Sirkin, Les, and Wiles, G.C., 1986, Surficial geologic map of New York: New York State Museum, Geological Survey, Map and Chart Series no. 40, Lower Hudson Sheet, scale 1:250:000.
Fisher, D.W., Isachsen, Y.W., and Richard, L.W., 1970, Geologic map of New York: New York State Museum and Science Service, Geological Survey, Map and Chart Series no. 15, Lower Hudson Sheet, scale 1:250,000.

Goldin, H.J., 1989, Waste not, want not - managing New York City's water supply: City of New York, Office of the Comptroller, $48 \mathrm{p}$.

Greeson, P.E., and Robison, F.L., 1970, Characteristics of New York lakes, part 1-Gazetteer of lakes, ponds, and reservoirs: U.S. Geological Survey Bulletin 68, 124 p.

Greeson, P.E., and Williams, G.E., 1970a, Characteristics of New York lakes, part 1A-Gazetteer of lakes, ponds, and reservoirs by counties: U.S. Geological Survey Bulletin 68A, $121 \mathrm{p}$. $1970 \mathrm{~b}$, Characteristics of New York lakes, part 1B-Gazetteer of lakes, ponds, and reservoirs by drainage basins: U.S. Geological Survey Bulletin 68B, $122 \mathrm{p}$.

New York State Department of Environmental Conservation, 1992, Inactive hazardous waste disposal sites in New York State, annual report, appendix volume 3-list of sites by county: New York State Department of Environmental Conservation, Division of Hazardous Waste Remediation.

Robideau, J.A., Burke, P.M., and Richard Lumia, 1984, Maximum known stages and discharges of New York State streams through September 1983: U.S. Geological Survey Open-File Report 83-927, 83 p.

U.S. Department of Commerce, Bureau of Census, 1990, 1990 census of population and housing: Public Law 94-171.

U.S. Geological Survey, 1971, Index of surface-water records to September 30, 1970, part 1.-North Atlantic Slope Basins: U.S. Geological Survey Circular 651, 89 p. 1984-92, Water resources data-New York, water years 1984-1992, volume 1, Eastern New York excluding Long Island: U.S. Geological Survey Water-Data Report (issued annually).

Wagner, L.A., 1982, Drainage areas of New York streams, by river basins - A stream gazetteer, part 1, data compiled as of October 1980: U.S. Geological Survey Open-File Report 81-1055, 359 p. 


\section{APPENDIX}

Geographic Information System (GIS) Data-Base coverages with description, scale, data type, source, and procedures used in development of each coverage used in selection of monitoring sites in the Croton Reservoir system.

Coverage: BASINS-USGS-delineated basin boundary of Croton Reservoir system

Scale: $1: 24,000$

Data type: Lines (Arcs)

Source agency: USGS

Coverage Development: The USGS New York drainage-area compilation (Wagner, 1982) is continually updated, and ARC/INFO coverages for all of New York State are being developed as part of this process. These coverages contain basin and subbasin boundaries, which are delineated by the USGS and checked by the Natural Resources Conservation Service.

Coverage: POPULATION-Population density

Scale: $1: 250,000$

Data type: Points

Source agency: U.S. Department of Commerce, 1990, Census of Population and Housing

Coverage Development: A point coverage of the 1990 Census of Population and Housing (U.S. Department of Commerce, 1990) for the conterminous United States was obtained, and the data for the study area were made into a separate coverage that includes the locations of population points retrieved at the "block group" summary level and the total number of persons and housing units represented by that point. Each point represents data from a polygon whose boundaries are not indicated in the coverage.

Coverage: LAND USE_Land use, divided into four major land-use categories: urban-commercial/industrial, urban-residential, rural-agriculture, and rural-undeveloped.

Scale: $1: 24,000$ and $1: 250,000$

Data type: Polygons

Source agency: Dutchess County and Connecticut: USGS; Putnam County: New York City Department of Environmental Protection (NYCDEP); Westchester County: Westchester County Planning Department

Coverage Development: Land-use data for Dutchess, Putnam, and Westchester Counties and Fairfield County, Conn., were obtained from different sources. Westchester County data were provided by the Westchester County Planning Department as an ARC/INFO coverage at a scale of 1:24,000, containing land-use data from the early 1980 's. This coverage indicated 13 different types of land use that, in this study, were combined into the four major land-use categories listed above. Putnam County data were provided by the NYCDEP as an ARC/INFO coverage of land use at a scale of 1:24,000. This coverage was interpreted from 1987 aerial photographs and updated in 1991 with field checking and represents about 50 land-use categories, which were combined into the four major land-use categories mentioned above. Dutchess County data and the parts of Fairfield, Conn., that lie in the study area were obtained from USGS 1:250,000 scale Geographic Information Retrieval and Analysis System (GIRAS), which represents data from the mid-1970's and includes more than 30 types of land use. These were combined into the four major land-use categories referred to above. After the land-use data for each county had been combined into the four categories, the separate coverages for each county were joined to create a single land-use coverage representing the entire study area. The boundaries of the land-use polygons were then checked to ensure matching across county and state boundaries. 
Coverage: ROADS - Transportation routes

Scale: $1: 24,000$

Data type: Lines (arcs)

Source agency: USGS

Coverage Development: Digital Line Graphs (DLGs) at 1:100,000 scale were obtained from the USGS and converted to an ARC/INFO coverage. The linework of this coverage was enhanced by digitizing some of the smaller roads from 1:24,000-scale planimetric maps provided by the New York State Department of Transportation.

Coverage: HYDROGRAPHY —Streams, aqueducts, and outlines of lakes and reservoirs

Scale: $1: 100,000$

Data type: Lines (Arcs)

Source agency: USGS

Coverage Development: Digital Line Graphs (DLGs) at a scale of 1:100,000 were obtained from the USGS and converted to an ARC/INFO coverage. The linework of this coverage was enhanced by digitizing smaller streams from 1:24,000-scale USGS topographic maps. In this coverage, wetlands were represented as lines through the center of the wetlands rather than by the boundaries of the wetlands.

Coverage: SURFACE-WATER GAGES-USGS streamflow and reservoir-stage-measuring sites

Scale: $1: 24,000$

Data type: Points

Source agency: USGS

Coverage Development: Data on active and inactive continuous streamflow-measurement sites were obtained through a retrieval from the USGS- National Water Information System (NWIS) data base. Data on miscellaneous streamflow-measurement sites were obtained from the following publications: Robideau and others (1984), U.S. Geological Survey (1971), Greeson and Robison (1970), Greeson and Williams (1970a,b), and annual USGS WaterData Reports (v. 1) for 1984-92 (NY-84, NY-85, NY-86, NY-87, NY-88, NY-89, NY-90, NY-91, and NY-92).

Data Set: INFLOWS AND DIVERSIONS - Diversions into and out of the Croton Reservoir system

Scale: $1: 24,000$

Data type: Table

Source agency: New York City Department of Environmental Protection (NYCDEP)

Coverage Development: A tabulation of information on the diversions into and out of the Croton Reservoir system, provided by the NYCDEP, included the losing body or receiving body of water, the amount of water and the party responsible for the diversion.

\section{Coverage: BEDROCK GEOLOGY}

Scale: $1: 250,000$

Data type: Polygons

Source agency: New York State Geological Survey (NYSGS)

Coverage Development: The bedrock geology of the study area was unavailable in digital format. Data from a map of the bedrock geology of New York State (Fisher and others, 1970, scale 1:250,000) was digitized into an ARC/ INFO coverage.

\section{Coverage: SURFICIAL GEOLOGY}

Scale: $1: 250,000$

Data type: Polygons

Source agency: New York State Geological Survey (NYSGS)

Coverage Development: The surficial geology of the study area was unavailable in digital format. Data from the New York State surficial geology map (Cadwell and others, 1986, scale 1:250,000) was digitized into an ARC/INFO coverage.

Coverage: PUBLIC-WATER-SUPPLY WITHDRAWALS-Public-supply well locations and associated well yields 
Scale: $1: 24,000$

Data type: Points

Source agency: USGS

Coverage Development: A retrieval was made from the USGS National Water Information System (NWIS) SiteSpecific Water-Use database (SSWUDS) to identify all the public-water supply withdrawal points within the study area. Information on each site included the name of the public-water supplier, the latitude and longitude of the withdrawal point, whether the withdrawal was from a surface-water supply or a ground-water supply, and the amount of water withdrawn. The data in the SSWUDS database were obtained from the New York State Department of Health and represent the year 1981.

Coverage: GROUND-WATER-WELLS-Locations and yields of wells finished in bedrock and stratified-drift well locations and yields (wells with yields greater than 25 gallons per minute)

Scale: $1: 24,000$

Data type: Points

Source agency: USGS

Coverage Development: A retrieval was made from the USGS National Water Information System (NWIS)

Ground-Water Site Inventory database (GWSI) to identify all the wells in the study area that had a reported yield of at least 25 gallons per minute. Information on each site included the identifying name, the latitude and longitude, the primary water use, depth of well, and yield of well. The type of aquifer (bedrock or surficial) tapped was interpreted from the well location, water use, and well depth.

Data Set: WATER-QUALITY POINT SOURCE—Site and type of possible point-source contamination, including State and Federal Superfund sites

Scale: $1: 24,000$

Data type: Tabulation

Source agency: New York State Department of Environmental Conservation (NYSDEC)

Coverage Development: A table was developed listing the location and type of possible point-source contamination within the study area. Site locations were obtained from the NYSDEC (1992).

Coverage: WATER QUALITY SITES-Site location, date, and type of water-quality analyses

Scale: $1: 24,000$

Data type: Points

Source agency: USGS and New York City Department of Environmental Protection (NYCDEP) computer data bases.

Coverage Development: A retrieval was made from the USGS National Water Information System (NWIS) Water-Quality database (QWDATA) to identify any USGS data-collection sites (ground water or surface water) in the study area at which water samples had been collected for chemical analysis. Information on these sites included the site name, whether it was a ground-water site or a surface-water site, the latitude and longitude, the number of samples collected and analyzed, the range of dates of sample collection, and the physical, chemical, and biological constituents for which each sample was analyzed. Also included in this coverage are the locations of surface-water sites run by the NYCDEP at which water samples have been collected and analyzed. Information from the NYCDEP on these sites includes the type of site (reservoir, stream, aqueduct), the location of the site, the number of samples collected, the frequency of sample collection, the period(s) during which the samples were collected, and the physical, chemical, and biological constituents for which for each sample was analyzed.

Coverage: DAMS - Locations of dams, including information on owner and body of water on which dam is located.

Scale: $1: 24,000$

Data type: Points

Source agency: New York State Department of Environmental Conservation (NYSDEC)

Coverage Development: A list of dams in New York State was obtained from the NYSDEC as a computer retrieval from the NYSDEC Dam Safety Project data base in July 1983. Included in the list are the dam name; the body of 
water impounded by the dam; the owner of the dam; and the stream, river, or reservoir on which the dam is located. Data on the dams in the study area were developed into an ARC/INFO point coverage.

Coverage: LAND SURFACE-Land-surface contours at 50-foot intervals

Scale: $1: 100,000$

Data type: Lines (Arcs)

Source agency: USGS

Coverage Development: Digital Elevation Models (DEMs) at 1:100,000 scale for the study area were obtained from the USGS and converted into an ARC/INFO coverage which, in turn, was modified to include only 50-foot landsurface contours and even multiples thereof. 